OPEN ACCESS

Edited by:

Yuji Morita,

Aichi Gakuin University, Japan

Reviewed by:

Paul Alan Hoskisson

University of Strathclyde, UK Claudio Avignone-Rossa,

University of Surrey, UK Jem Stach,

University of Newcastle, UK

*Correspondence: Bey-Hing Goh goh.bey.hing@monash.edu: Learn-Han Lee lee.learn.han@monash.edu; leelearnhan@yahoo.com

Specialty section: This article was submitted to Antimicrobials, Resistance and Chemotherapy,

a section of the journal

Frontiers in Microbiology

Received: 23 December 2015 Accepted: 29 March 2016

Published: 22 April 2016

Citation:

Ser H-L, Law JW-F, Chaiyakunapruk $N$, Jacob SA, Palanisamy UD, Chan K-G, Goh B-H and Lee L-H (2016) Fermentation Conditions that Affect

Clavulanic Acid Production in

Streptomyces clavuligerus: A Systematic Review.

Front. Microbiol. 7:522.

doi: 10.3389/fmicb.2016.00522

\section{Fermentation Conditions that Affect Clavulanic Acid Production in Streptomyces clavuligerus: A Systematic Review}

\author{
Hooi-Leng Ser 1,2, Jodi Woan-Fei Law ${ }^{1,2}$, Nathorn Chaiyakunapruk 1,3,4,5, \\ Sabrina Anne Jacob ${ }^{1}$, Uma Devi Palanisamy ${ }^{2}$, Kok-Gan Chan ${ }^{6}$, Bey-Hing Goh ${ }^{1,2,7 *}$ and \\ Learn-Han Lee ${ }^{1,2,7 *}$
}

${ }^{1}$ School of Pharmacy, Monash University Malaysia, Bandar Sunway, Malaysia, ${ }^{2}$ Biomedical Research Laboratory, Jeffrey Cheah School of Medicine and Health Sciences, Monash University Malaysia, Bandar Sunway, Malaysia, ${ }^{3}$ Department of Pharmacy Practice, Faculty of Pharmaceutical Sciences, Center of Pharmaceutical Outcomes Research, Naresuan University, Phitsanulok, Thailand, ${ }^{4}$ School of Pharmacy, University of Wisconsin-Madison, Madison, WI, USA, ${ }^{5}$ School of Population Health, University of Queensland, Brisbane, QLD, Australia, ${ }^{6}$ Division of Genetics and Molecular Biology, Faculty of Science, Institute of Biological Sciences, University of Malaya, Kuala Lumpur, Malaysia, ${ }^{7}$ Center of Health Outcomes Research and Therapeutic Safety (Cohorts), School of Pharmaceutical Sciences, University of Phayao, Phayao, Thailand

The $\beta$-lactamase inhibitor, clavulanic acid is frequently used in combination with $\beta$-lactam antibiotics to treat a wide spectrum of infectious diseases. Clavulanic acid prevents drug resistance by pathogens against these $\beta$-lactam antibiotics by preventing the degradation of the $\beta$-lactam ring, thus ensuring eradication of these harmful microorganisms from the host. This systematic review provides an overview on the fermentation conditions that affect the production of clavulanic acid in the firstly described producer, Streptomyces clavuligerus. A thorough search was conducted using predefined terms in several electronic databases (PubMed, Medline, ScienceDirect, EBSCO), from database inception to June 30th 2015. Studies must involve wild-type Streptomyces clavuligerus, and full texts needed to be available. A total of 29 eligible articles were identified. Based on the literature, several factors were identified that could affect the production of clavulanic acid in S. clavuligerus. The addition of glycerol or other vegetable oils (e.g., olive oil, corn oil) could potentially affect clavulanic acid production. Furthermore, some amino acids such as arginine and ornithine, could serve as potential precursors to increase clavulanic acid yield. The comparison of different fermentation systems revealed that fed-batch fermentation yields higher amounts of clavulanic acid as compared to batch fermentation, probably due to the maintenance of substrates and constant monitoring of certain entities (such as $\mathrm{pH}$, oxygen availability, etc.). Overall, these findings provide vital knowledge and insight that could assist media optimization and fermentation design for clavulanic acid production in S. clavuligerus.

Keywords: clavulanic acid, clavulanate, Streptomyces clavuligerus, fermentation, systematic review 


\section{INTRODUCTION}

Microorganisms serve as attractive resources, owing to their ability to synthesize structurally-diverse substances with various bioactivities (Demain, 1999; Newman et al., 2000; Bérdy, 2005; Demain and Sanchez, 2009). These microbial natural products may be used as effective drug(s) or act as drug lead compounds that could be further modified and developed for higher efficacy. Within the Bacteria domain, actinomycetes showed unprecedented ability to produce potentially novel, clinically useful, secondary metabolites such as anticancer, antioxidants, antivirals and antibacterials (Ara et al., 2014; Lee et al., 2014a,b; Manivasagan et al., 2014; Azman et al., 2015; Ser et al., 2015a,b; Tan et al., 2015). These filamentous bacteria produce around 8700 antibiotics, with the majority of them derived from members of the Streptomyces genus (Bérdy, 2005; Demain and Sanchez, 2009; de Lima Procópio et al., 2012). As the largest antibioticproducing genus, Streptomyces species are capable of producing different classes of antibiotics including aminoglycosides (e.g., streptomycin by $S$. griseus), macrolides (e.g., tylosin from $S$. fradiae), and $\beta$-lactams (e.g., cephamycin and clavulanic acid by S. clavuligerus) (Waksman et al., 1944; Brown et al., 1976; Reading and Cole, 1977; Okamoto et al., 1982).

The $\beta$-lactam antibiotics are one of the most popular classes of antibacterial agents, whose mechanism of action is via inhibition of bacterial cell wall synthesis (Page, 2012). Soon after the utilization of $\beta$-lactam antibiotics, a number of bacteria have been found to exhibit resistance to this class of drugs. One of the strategies deployed by this group of bacteria to survive against $\beta$-lactam antibiotics is by the production of a $\beta$-lactamhydrolyzing enzyme - $\beta$-lactamase; which functions to neutralize these antibiotics by cleaving the $\beta$-lactam ring (Wilke et al., 2005; Toussaint and Gallagher, 2015). Thus, to overcome this resistance, $\beta$-lactamase inhibitors are often used in conjunction with $\beta$-lactam antibiotics as these compounds prevent the degradation of these antibiotics and increase the efficacy of these drugs (Saudagar et al., 2008).

Clavulanic acid was first purified as a novel $\beta$-lactamase inhibitor from S. clavuligerus ATCC 27064, which was isolated from South American soil in 1971 (Higgens and Kastner, 1971; Brown et al., 1976). This compound presents with a nucleus similar to that of penicillin, with notable differences such as lacking anacylamino side chain, containing oxygen in place of sulfur, and having a $\beta$-hydroxyethylidine substituent in the oxazolidine ring (Brown et al., 1976; Saudagar et al., 2008). Clavulanic acid or clavulanate, is commercially used along with amoxicillin (Augmentin) and this combination has been listed as an important antibacterial agents in the WHO list of essential medicines (2015) (Toussaint and Gallagher, 2015). This compound was first recovered from the fermentation process, which remains as one of the most frequently used strategies to manufacture important drugs and their intermediates for medicinal use. In order to facilitate the higher production of valuable compound as such, advanced fermentation technologies were subsequently developed, which included fed-batch fermentation systems (Thiry and Cingolani, 2002; Schmidt, 2005).
At the same time, researchers began to look into the biosynthesis pathway of clavulanic acid in an attempt to maximize its production (Figure 1). These efforts then resulted in the identification of two important precursors for clavulanic acid-arginine (C5 precursor) and glutaraldehyde-3-phosphate (C3 precursor) (Romero et al., 1986; Kanehisa and Goto, 2000; Kanehisa et al., 2016). Apart from clavulanic acid, S. clavuligerus is known to produce other clavams and cephamycin; as illustrated in Figure 1. As S. clavuligerus is unable to assimilate glucose, various compounds have been studied as $\mathrm{C} 3$ precursor candidates to ensure proper formulation of fermentation media and improve the yield of clavulanic acid (Aharonowitz and Demain, 1978; Garcia-Dominguez et al., 1989; Pérez-Redondo et al., 2010). Thus, this systematic review examined the effect of different fermentation conditions on the production of clavulanic acid in S. clavuligerus. Based on the available literature, our objective was to describe how additional supplements in basal medium, $\mathrm{pH}$, as well as temperature affect the production of the $\beta$ lactamase inhibitor in $S$. clavuligerus, which in turn could assist and improve the development of fermentation media and/or systems for the production of this valuable antibiotic, clavulanic acid.

\section{METHODS}

This systematic review was carried out in accordance with the preferred reporting items for systematic reviews and metaanalyses (PRISMA) guidelines (Moher et al., 2009).

\section{Database Search}

Systematic searches were performed in the following databases: PubMed, Medline, ScienceDirect, and EBSCO. MeSH terms were "Streptomyces," "clavuligerus" combined with "clavulanic acid" or "clavulanate." We included studies from database inception to June 30th, 2015.

\section{Study Selection and Data Extraction}

Two reviewers (H-LS and JW-FL) independently screened and evaluated all titles and abstracts retrieved from the comprehensive search, based on the inclusion and exclusion criteria. The bibliographies of relevant studies were checked for additional publications. Full text of selected original articles were then obtained and reviewed. Any disagreements between the two reviewers were resolved by consensus. Studies providing data of clavulanic acid production in Streptomyces clavuligerus were included. Other inclusion criteria were: (1) studies must involve wild-type Streptomyces clavuligerus; (2) studies must describe fermentation conditions using batch and/or fedbatch fermentation strategies; and (3) studies must report the specific amount of clavulanic acid produced by Streptomyces clavuligerus. Studies conducted using S. clavuligerus mutants, studies conducted in organisms other than S. clavuligerus, and studies reporting only specific production of clavulanic acid; were excluded. We also excluded solid phase extraction studies, immobilization studies, and all reviews, conference abstracts, systematic reviews, meta-analyses, comments, and letters to the editor. The following information was extracted independently 


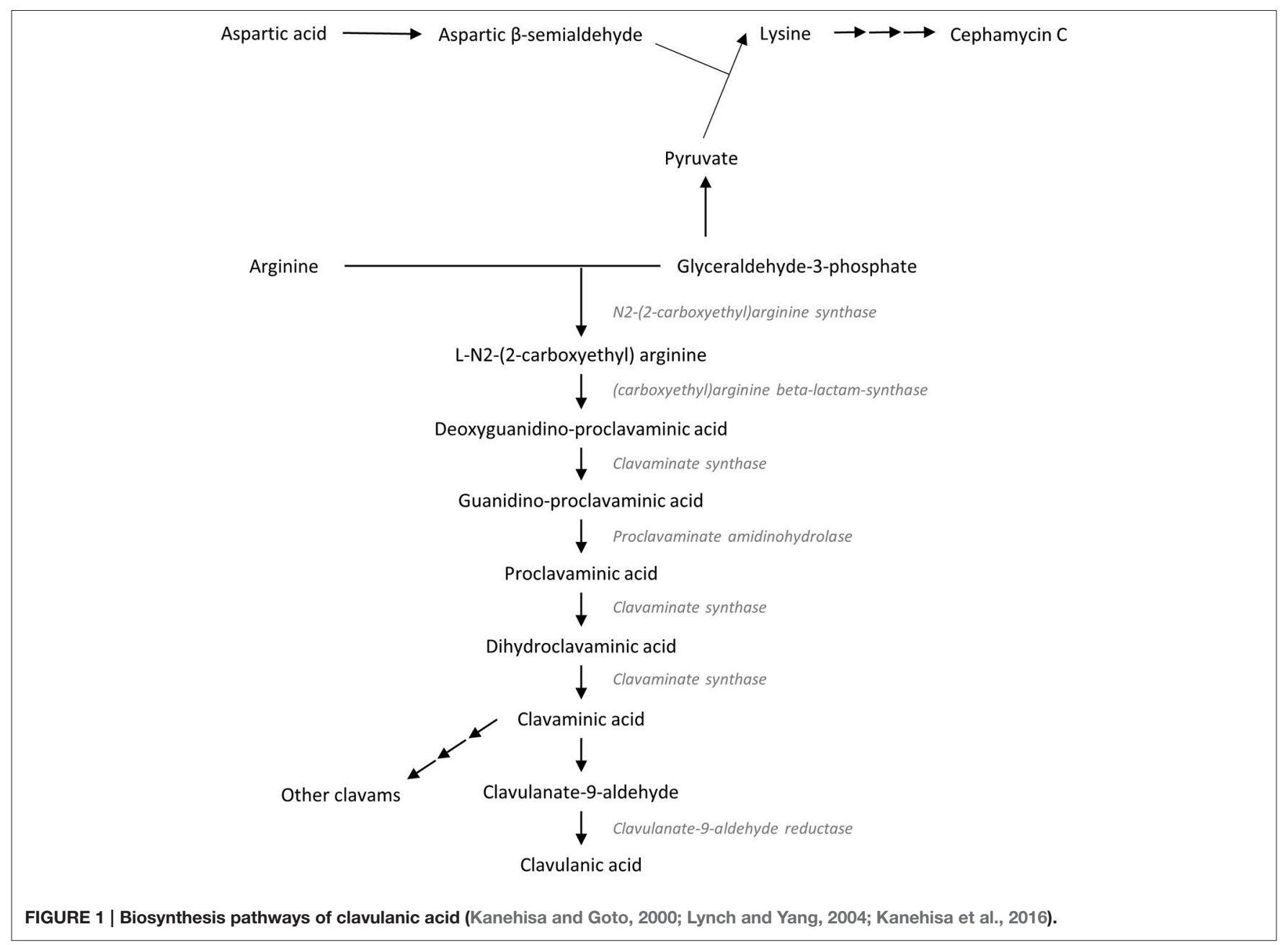

by the two reviewers from each study (Table 1): (1) study and year of publication, (2) fermentation type and volume, (3) oxygen control and/or airflow control, (4) fermentation/production media composition and $\mathrm{pH},(5)$ addition of supplements (e.g., glycerol, starch, and vegetable oils), and (6) maximum clavulanic acid produced. Any discrepancies were discussed between both authors.

\section{RESULTS}

\section{Literature Search}

The search yielded a total of 627 articles, while an additional 11 articles were obtained from other sources (Figure 2). After the removal of duplicate records, a total of 474 articles were accessed, out of which 432 articles were excluded based on their titles and abstracts. 42 full text articles were reviewed, out of which 29 studies were eligible for the qualitative analysis according to the inclusion criteria (Table 1). The analysis was divided into several categories based on the design of the experiments: (a) utilization of glycerol or starch as sole carbon source, (b) addition of glycerol and different oil in batch fermentation, (c) amino acids as supplements in basal medium, (d) other factors affecting clavulanic acid production in batch fermentation, and (e) comparison between batch and fed-batch fermentations.

\section{Utilization of Glycerol, Starch, or Sucrose as Sole Carbon Source}

Thakur et al. (1999) and Maranesi et al. (2005) described that different media compositions resulted in different levels of clavulanic acid production. When comparing between glycerol and sucrose as a sole carbon source, Lee and Ho (1996) observed no production of clavulanic acid in media added with glycerol, but higher production of the compound in media with sucrose $(3.63 \mathrm{mg} / \mathrm{L})$. Similar findings were also reported by Ives and Bushell (1997) where no production of clavulanic acid was observed in glycerol-containing C-limited media. Meanwhile, another study by Thakur et al. (1999) demonstrated that the addition of dextrin or glycerol as a sole carbon source neither improved nor decreased the production of clavulanic acid. On the contrary, two studies reported a totally different observationbasal media containing glycerol exhibited higher maximum amounts of clavulanic acid as compared to starch (Saudagar and Singhal, 2007a; Bellão et al., 2013). Indeed the maximum amount of clavulanic acid observed in media containing glycerol 


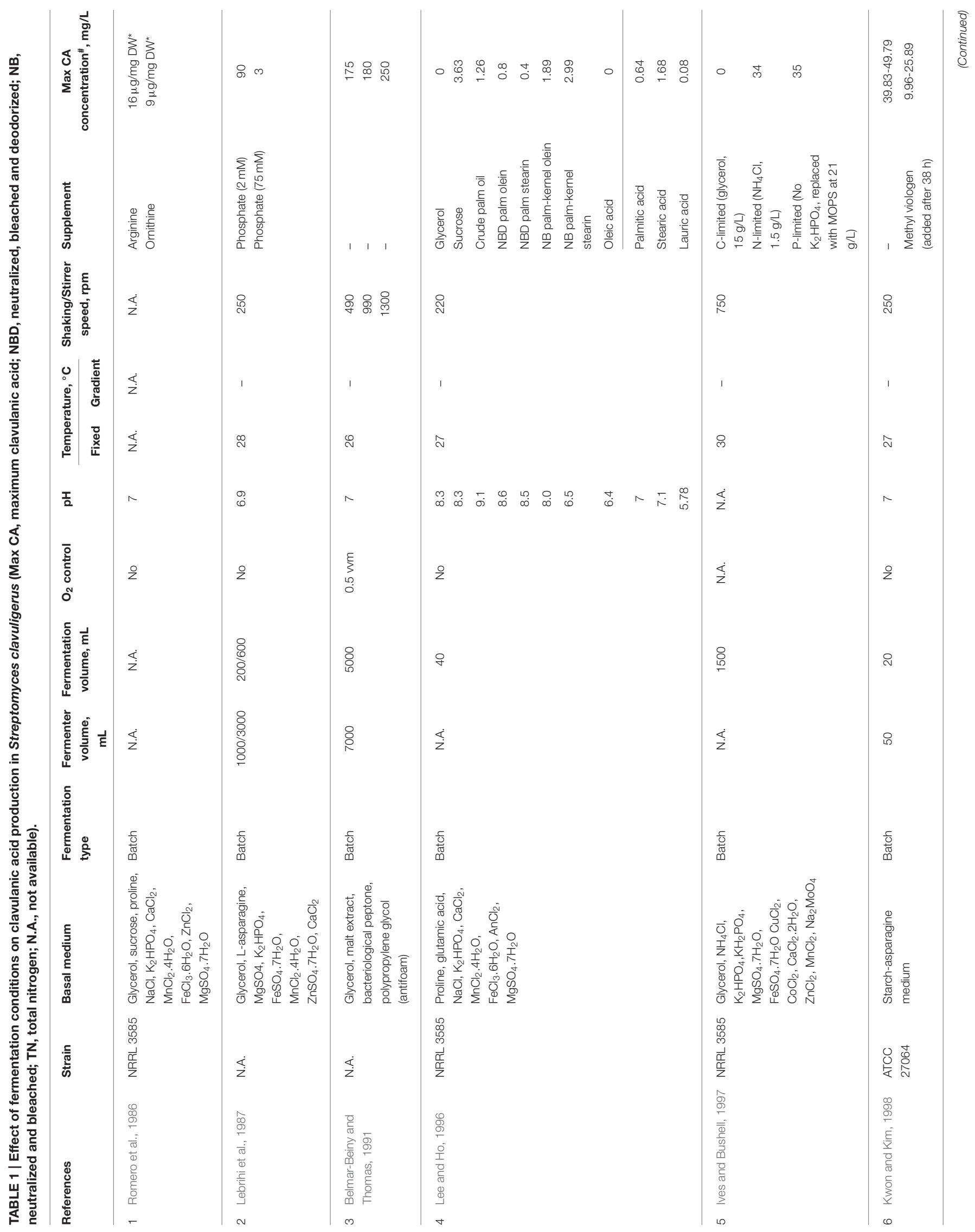




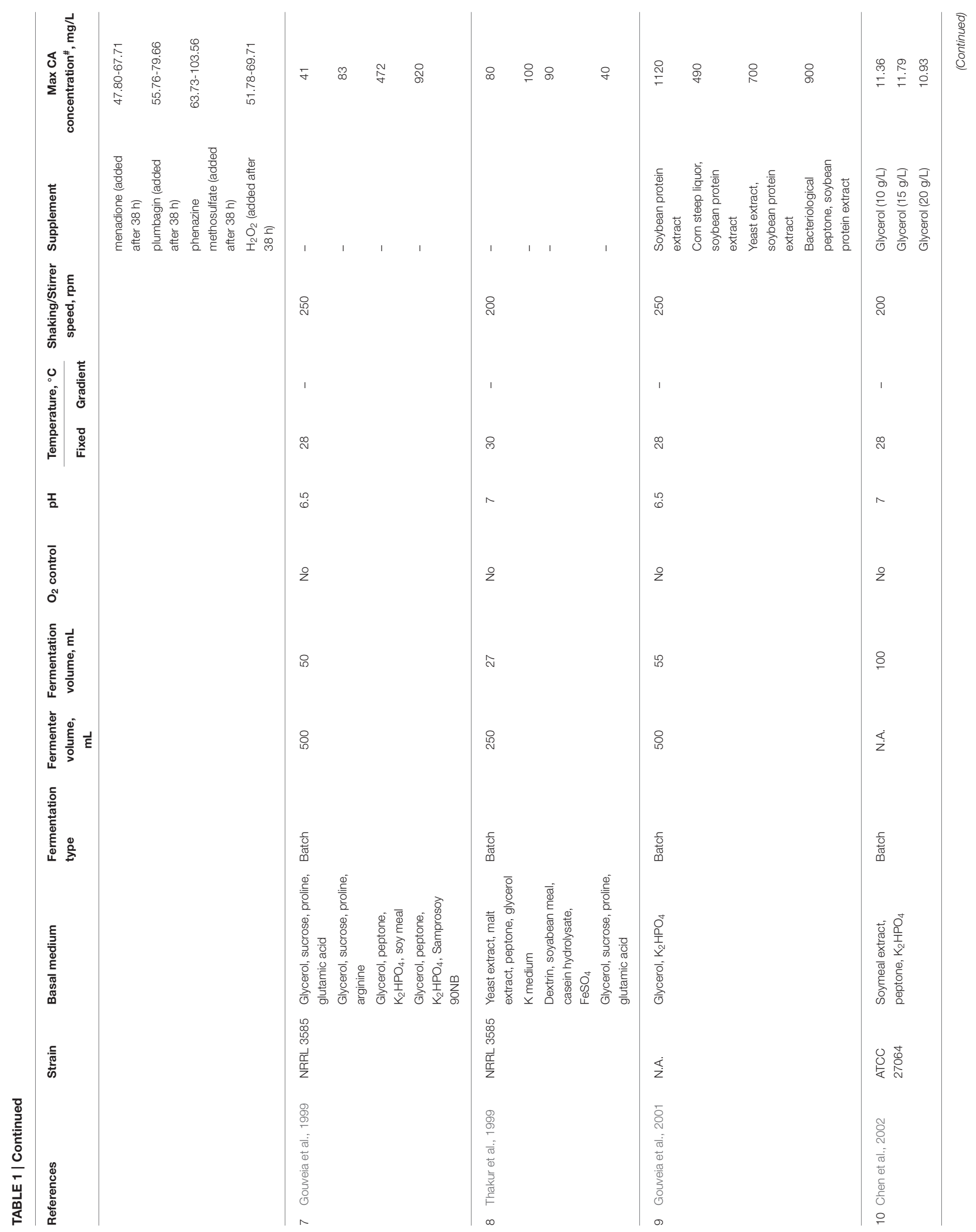




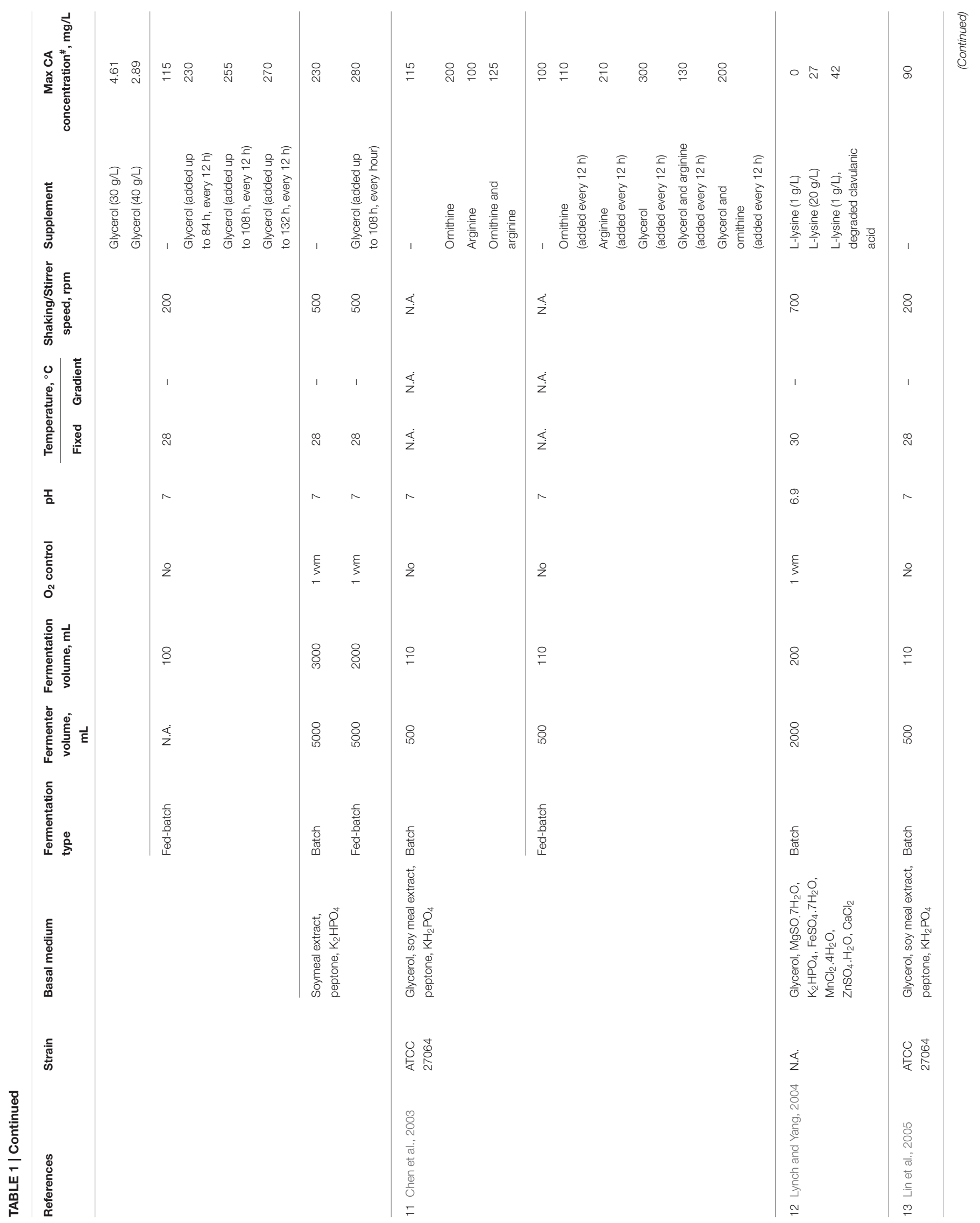




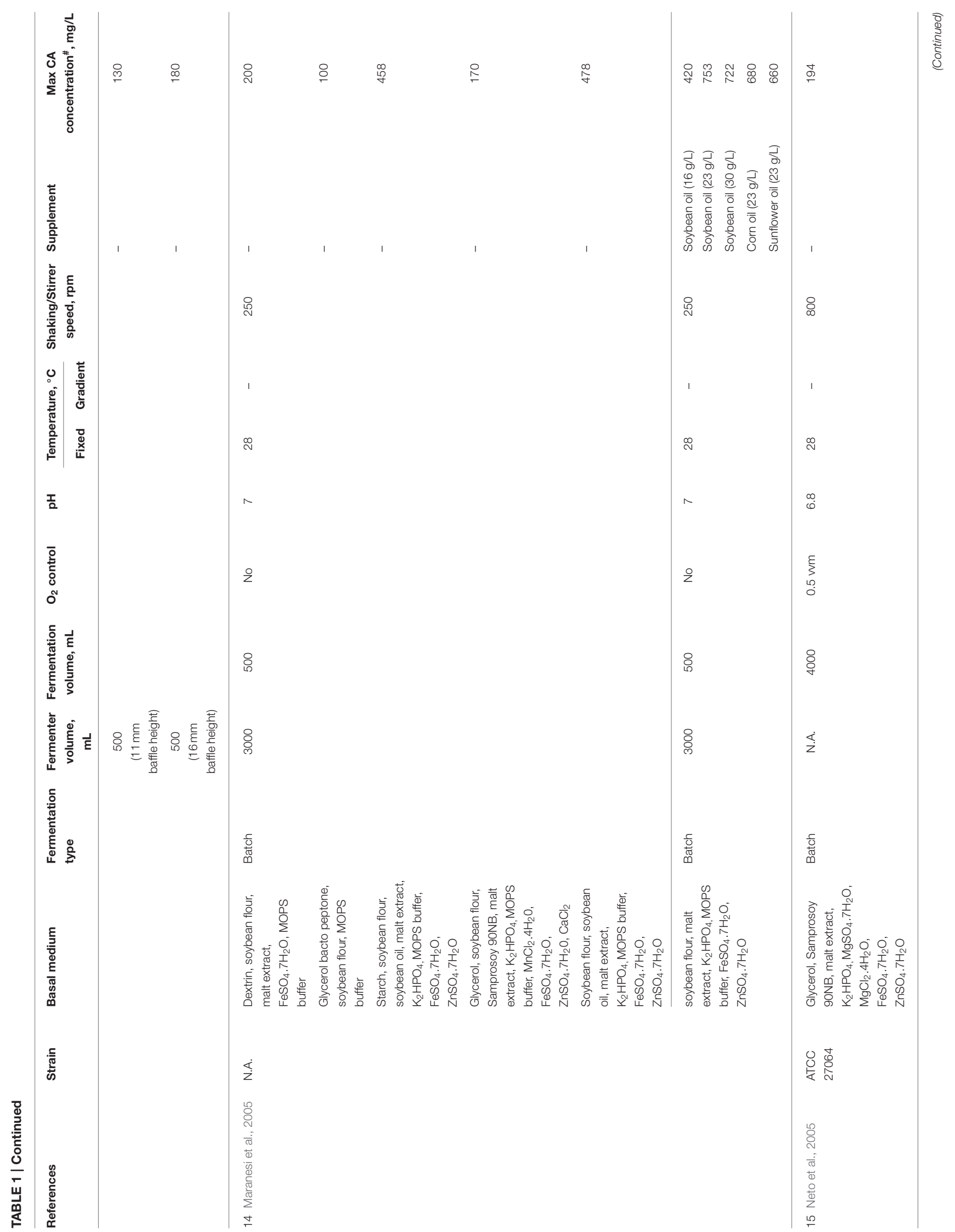




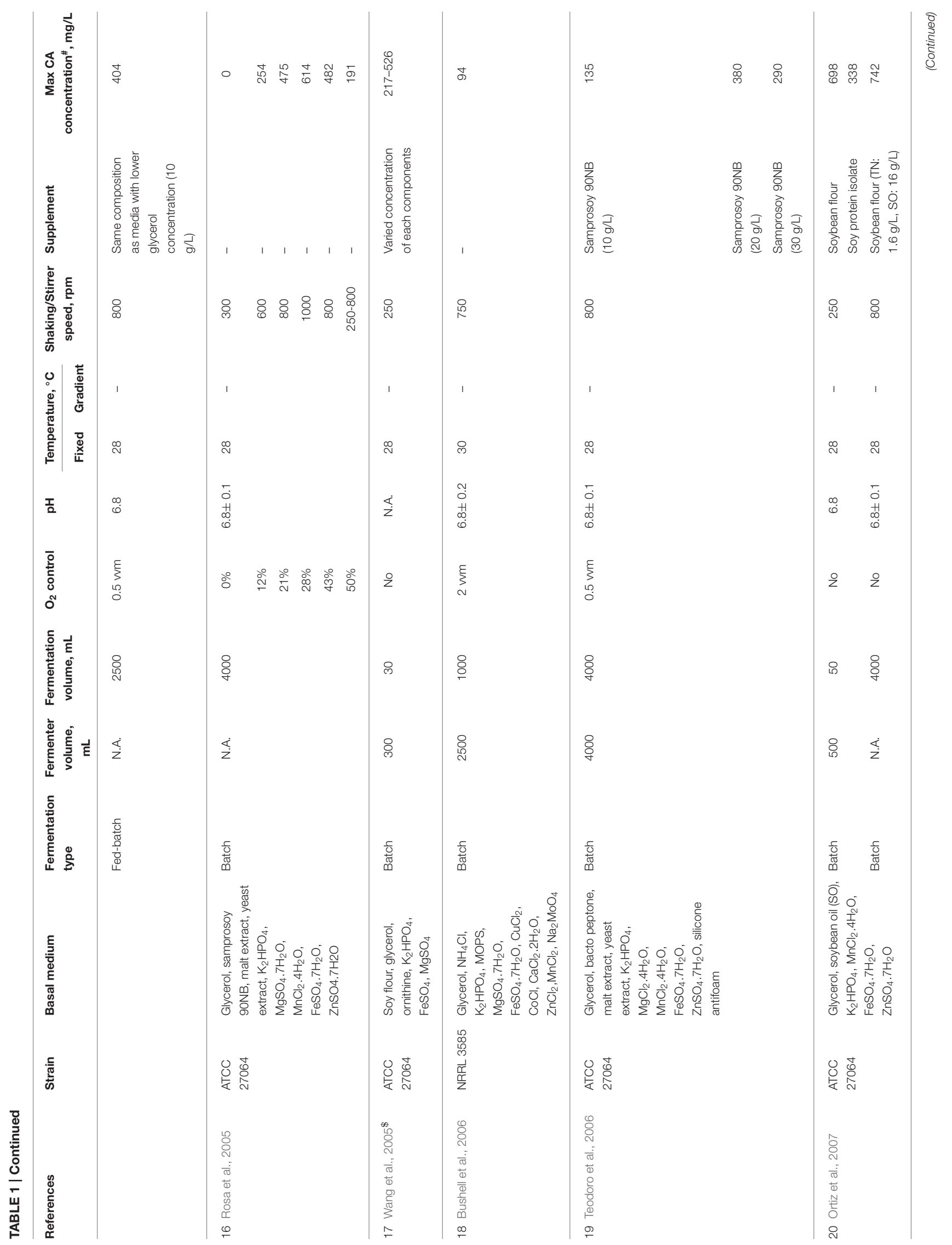




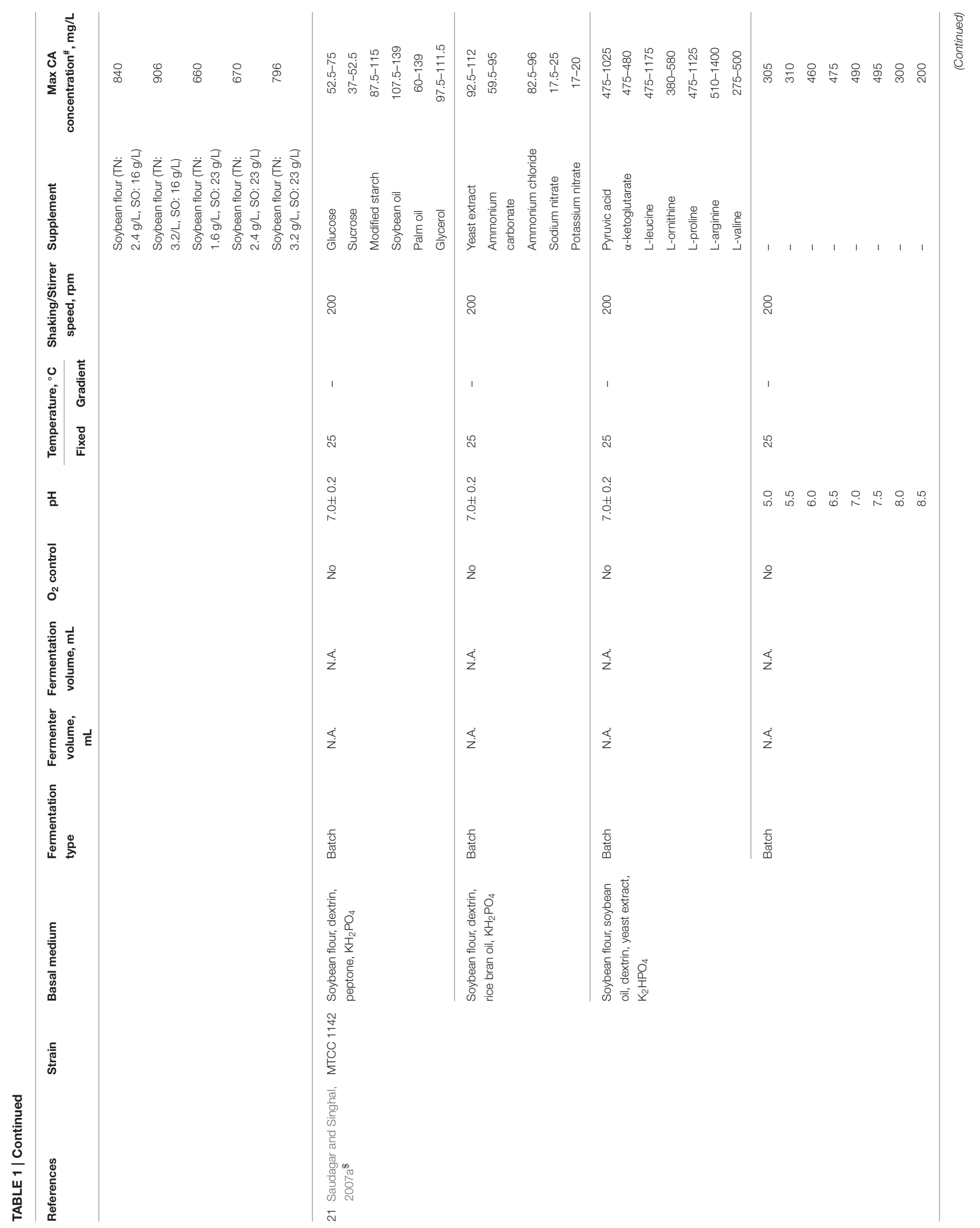




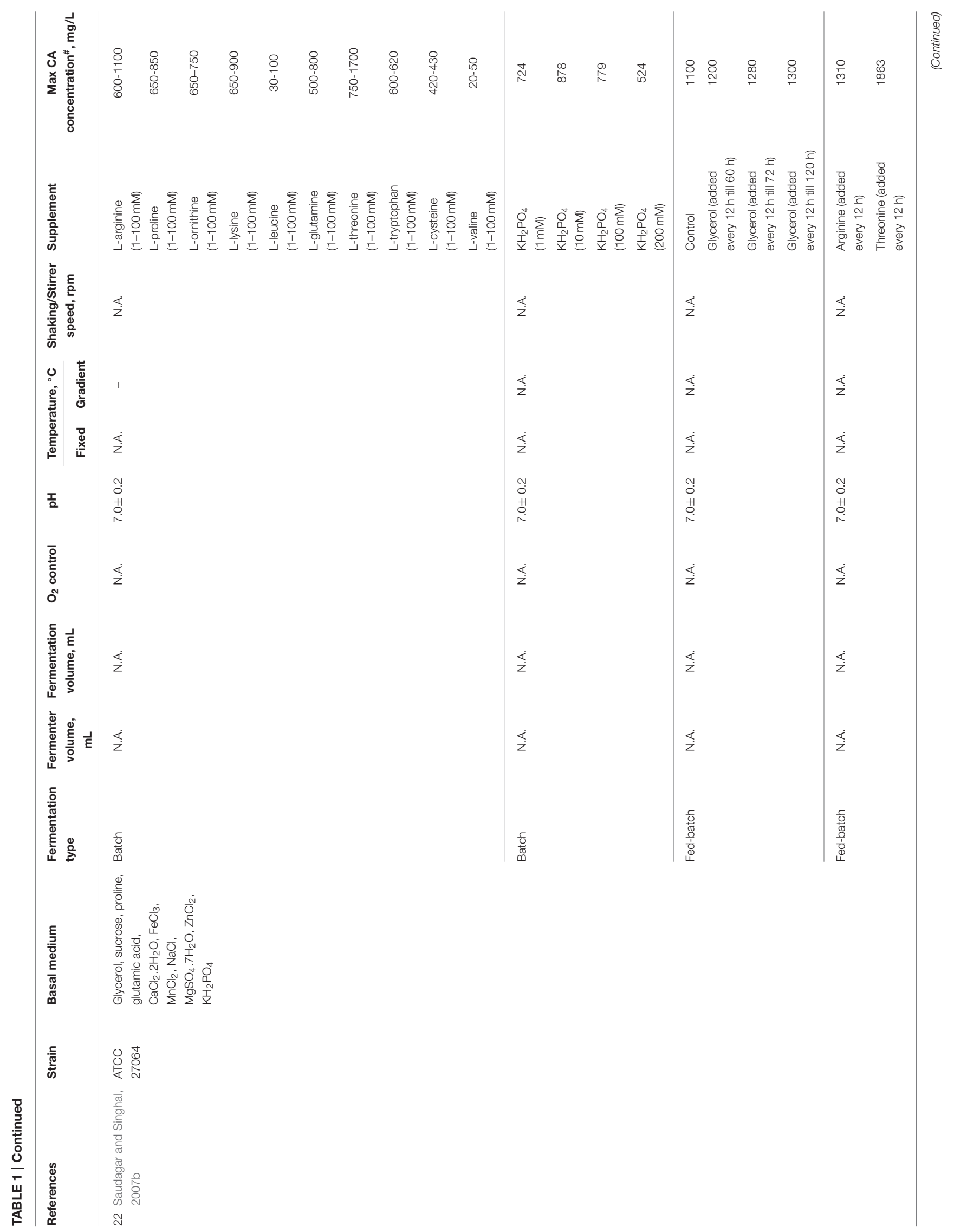




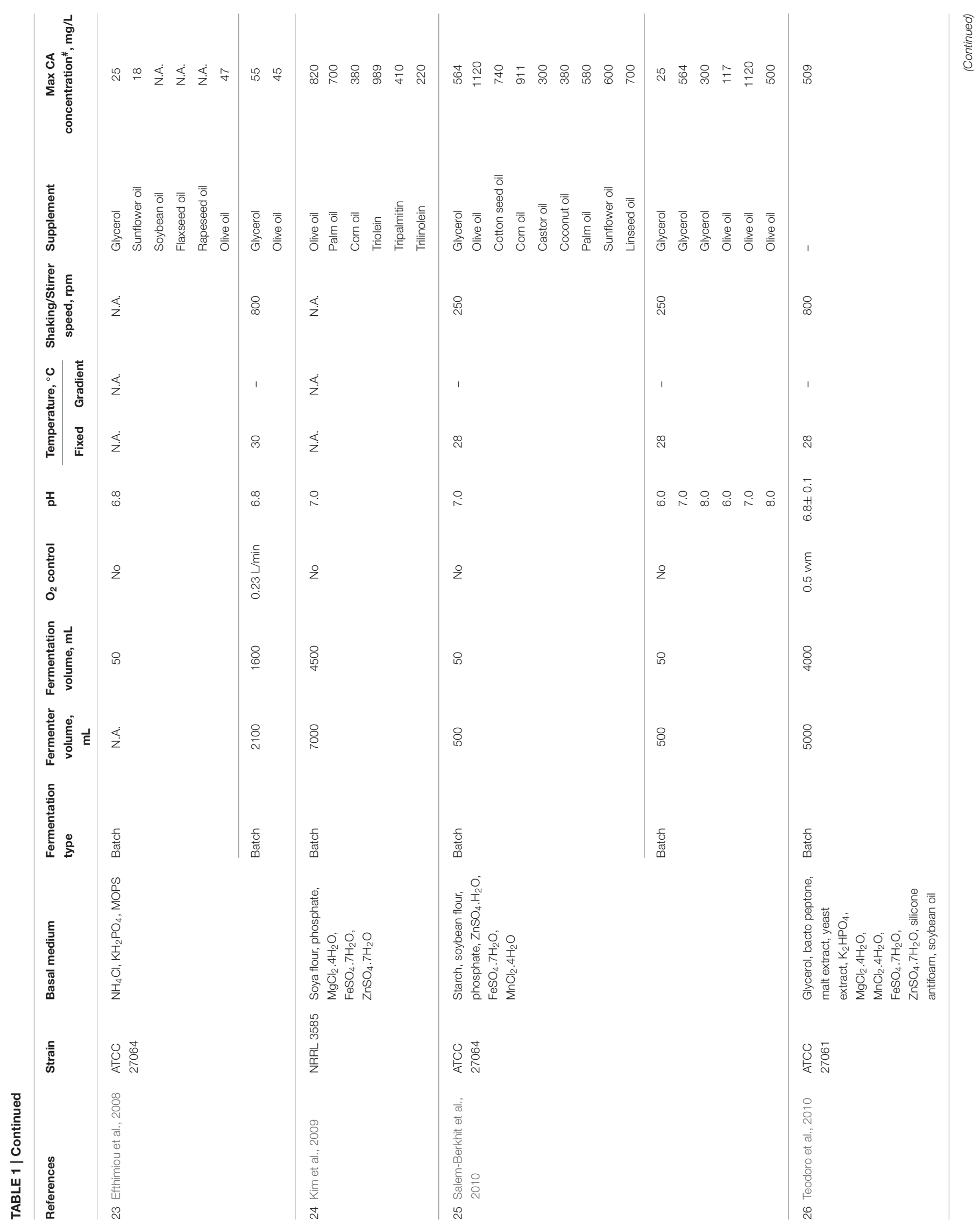




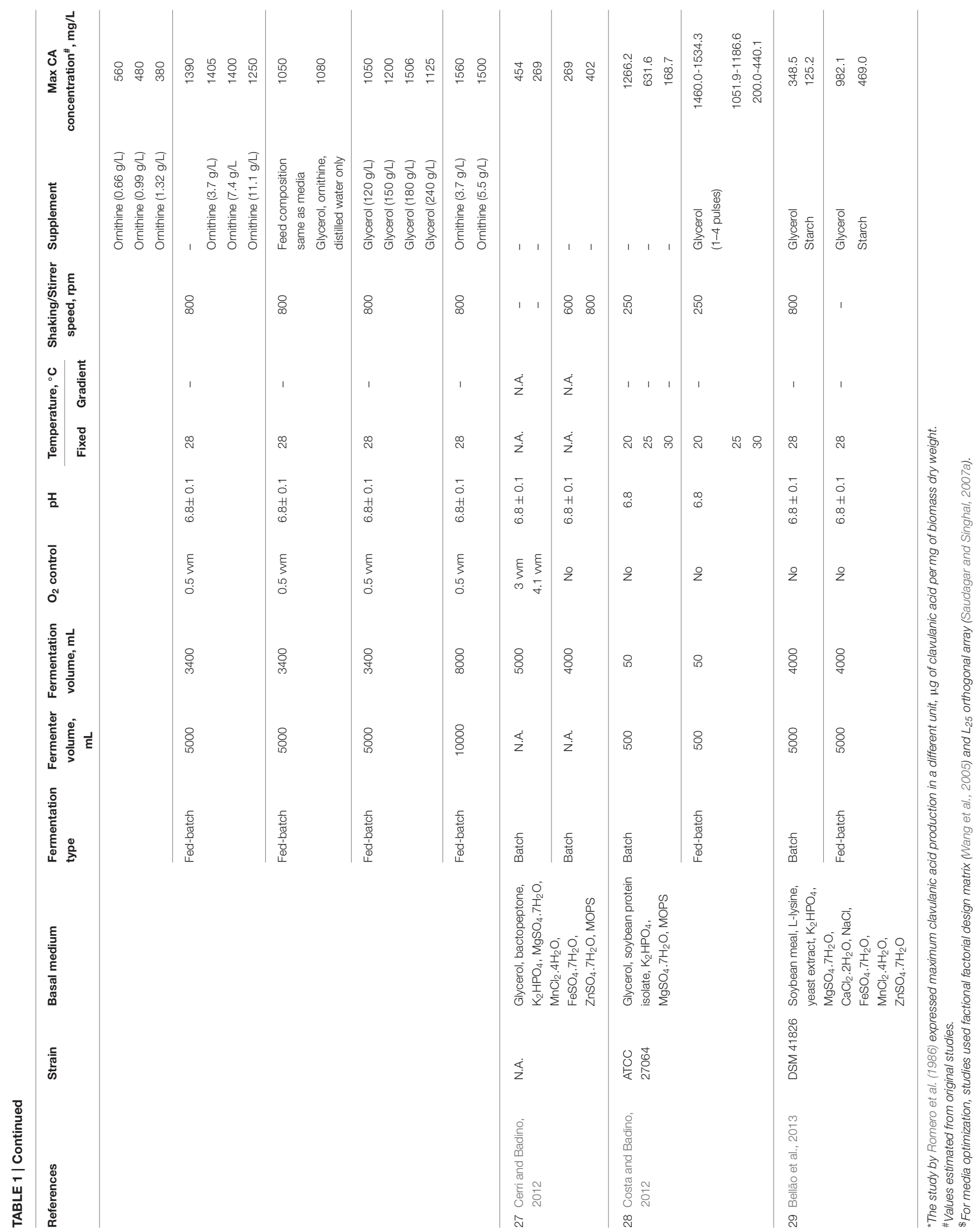




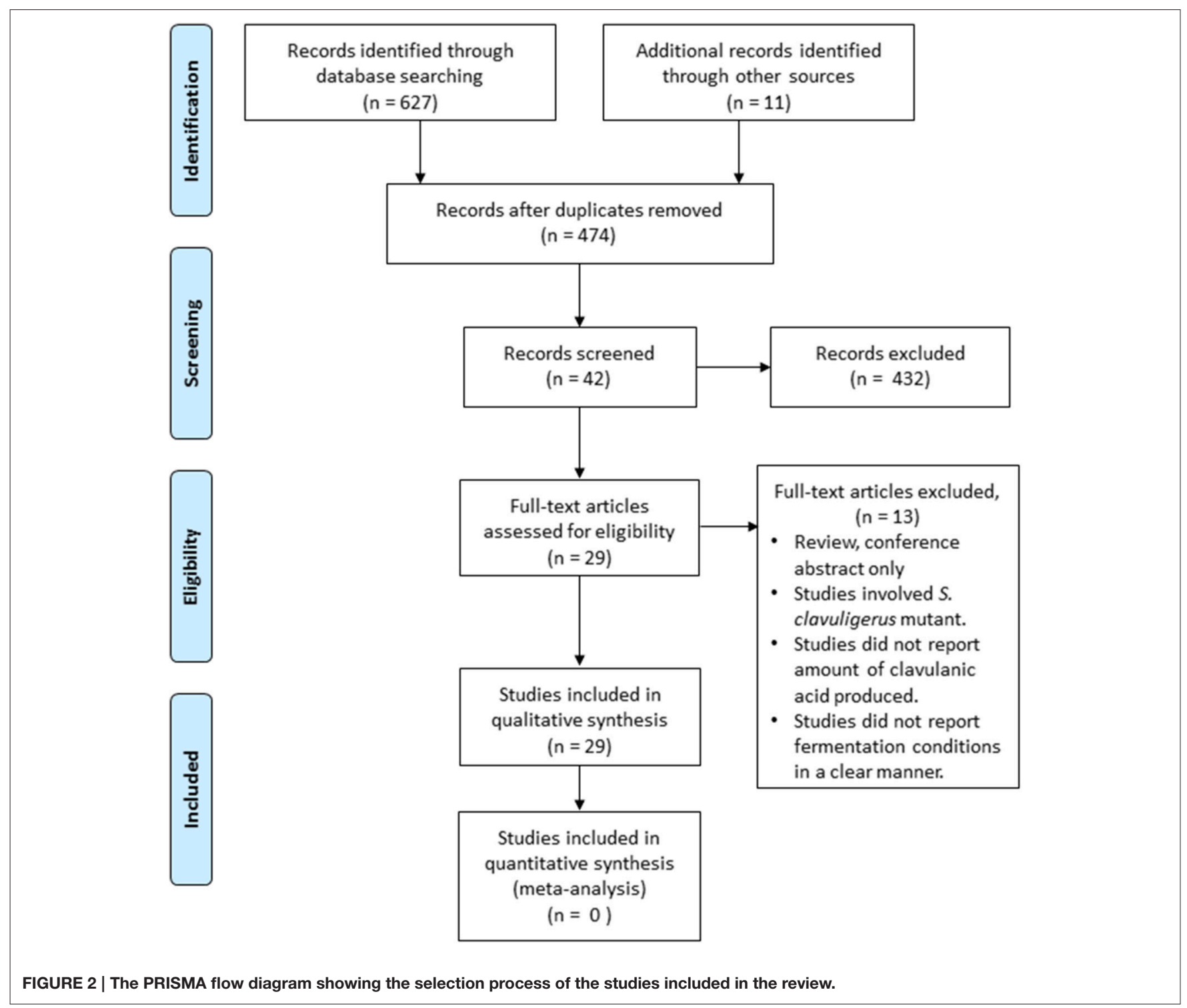

was found to be $348.5 \mathrm{mg} / \mathrm{L}$, nearly two times higher compared to media with starch as a sole carbon source (Bellão et al., 2013). Additionally, two other studies by Chen et al. (2002) and Saudagar and Singhal (2007a) revealed a biphasic dose response of glycerol; whereby clavulanic acid production was inhibited at concentrations which were either too high or too low.

\section{Addition of Glycerol and Different Oil in Batch Fermentation}

Apart from glycerol, several studies $(n=6)$ have also looked at how other oil and unsaturated fatty acids affect the production of clavulanic acid in S. clavuligerus. An earlier study revealed a relatively low production of clavulanic acid in media containing different fractions of palm oil or its purified, major constituents (e.g., palmitic acid, stearic acid, lauric acid, oleic acid) (Lee and Ho, 1996). A more recent study in 2009 by Kim et al. (2009) reported maximum clavulanic acid production of $700 \mathrm{mg} / \mathrm{mL}$ in media supplemented with palm oil, an intermediate value as compared to other oil sources. The highest clavulanic acid production reported in the same study was observed in media containing triolein (which is a major constituent of palm oil) at $989 \mathrm{mg} / \mathrm{L}$.

Different vegetable oils may stimulate the production of clavulanic acid, as demonstrated by two different studies. In batch fermentation, Efthimiou et al. (2008) described increased clavulanic acid production when olive oil was used in place of glycerol as a sole carbon source; with a maximum concentration of $47 \mathrm{mg} / \mathrm{L}$ being recorded, which is nearly double that observed in media containing glycerol $(25 \mathrm{mg} / \mathrm{L})$. These results were consistent with another recent study, whereby the addition of olive oil improved clavulanic acid production as compared to glycerol; with a maximum concentration at 1120 and $564 \mathrm{mg} / \mathrm{L}$, respectively (Salem-Berkhit et al., 2010). Relatively high production was also observed in media supplemented with 
corn oil $(911 \mathrm{mg} / \mathrm{L})$, followed by cotton seed oil $(740 \mathrm{mg} / \mathrm{L})$, and linseed oil $(700 \mathrm{mg} / \mathrm{L})$. Media with castor oil was found to yield the lowest amount of clavulanic acid $(300 \mathrm{mg} / \mathrm{L})$. Given that different vegetable oils may have a slight difference in fatty acids and lipid composition, Maranesi et al. (2005) described that when the same concentration of oil was used, the production of clavulanic acid differed slightly between soybean oil, corn oil, and sunflower oil. The greatest concentrations of clavulanic acid using soybean oil, corn oil, and sunflower oil was recorded to be between the ranges of $660-753 \mathrm{mg} / \mathrm{L}$. These results were consistent with a study by Saudagar and Singhal (2007a), as similar concentrations of clavulanic acid was observed in media containing palm oil and soybean oil.

\section{Protein and Amino Acids as Supplements in Basal Medium}

The choice of soybean flour or soy protein isolate in fermentation media affects the production of clavulanic acid by $S$. clavuligerus (Gouveia et al., 1999; Wang et al., 2005; Ortiz et al., 2007). The difference in the type of protein as a source of nitrogen was found to affect the production of clavulanic acid as studied by Gouveia et al. (2001). Increased production of clavulanic acid was reported in media containing soybean flour $(698 \mathrm{mg} / \mathrm{L})$ rather than soy protein isolate $(338 \mathrm{mg} / \mathrm{L})$. Further investigation with soybean flour revealed that different amounts of nitrogen with varied amounts of soybean oil, produced different concentrations of clavulanic acid $(660 \mathrm{mg} / \mathrm{L}-906 \mathrm{mg} / \mathrm{L})$. Similar findings were reported by Teodoro et al. (2006) as different clavulanic acid concentrations were observed $(135-380 \mathrm{mg} / \mathrm{L})$ in media containing varied concentrations of soy protein isolate.

The investigation on the role of amino acids as a source of nitrogen in the production of clavulanic acid began in 1986 (Romero et al., 1986). Several studies focusing on the effects of the amino acids, arginine and ornithine; toward the production of clavulanic acid showed inconsistent results. Romero et al. (1986) reported maximum concentrations of clavulanic acid in a slightly different manner, where $16 \mu \mathrm{g} /$ dry weight of biomass was observed with arginine; while $9 \mu \mathrm{g} / \mathrm{dry}$ weight of biomass was observed with ornithine. Differing results were recorded by Chen et al. (2003) as media supplemented with ornithine contained higher concentrations of clavulanic acid at $200 \mathrm{mg} / \mathrm{L}$, compared with arginine at $100 \mathrm{mg} / \mathrm{L}$. Nevertheless, recent studies supported the results of Romero et al. (1986); where higher amounts of clavulanic acid were seen in media supplemented with different concentrations of arginine compared to ornithine (Saudagar and Singhal, 2007a,b). One of the studies also tested the effect of other amino acids such as L-proline, Llysine, L-leucine, L-glutamine, L-threonine, L-tryptophan, Lcysteine, and L-valine (Saudagar and Singhal, 2007b). Among these amino acids, the highest concentration of clavulanic acid was observed in media supplemented with L-threonine; which was not reported in other literature included in this systematic review. Lynch and Yang (2004) tested the influence of L-lysine further by adding degraded clavulanic acid into the fermentation broth. The study suggested that L-lysine is one of the most important amino acids for the production of clavulanic acid, given that fermentation broths with low concentrations of L-lysine $(1 \mathrm{~g} / \mathrm{L})$ failed to yield any clavulanic acid. Moreover, the addition of degraded clavulanic acid showed improved clavulanic acid production (maximum clavulanic acid concentration at $42 \mathrm{mg} / \mathrm{L}$ ) as compared to fermentation broths with L-lysine alone $(20 \mathrm{~g} / \mathrm{L}$, clavulanic acid concentration at $27 \mathrm{mg} / \mathrm{L}$ ).

\section{Other Factors Affecting Clavulanic Acid Production in Batch Fermentation}

Aside from sole carbon or nitrogen sources, other factors that may affect the production of clavulanic acid include the addition of phosphate, $\mathrm{pH}$, temperature, and agitation or shaking speed. The potential repressive effect of phosphate on clavulanic acid production in S. clavuligerus was demonstrated by two selected studies (Lebrihi et al., 1987; Bushell et al., 2006; Saudagar and Singhal, 2007b). The study by Lebrihi et al. (1987) tested two levels of phosphate, 2 and $75 \mathrm{mM}$. Based on HPLC measurements, the lower concentration of phosphate $(2 \mathrm{mM})$ in fermentation media was found to contain significantly higher levels of clavulanic acid; with maximum concentration observed at $90 \mathrm{mg} / \mathrm{L}$, as compared to $3 \mathrm{mg} / \mathrm{L}$ observed in fermentation media containing high concentrations of phosphate $(75 \mathrm{mM})$. Without changing the $\mathrm{pH}$ of the media, Saudagar and Singhal (2007b) demonstrated that the addition of phosphate in the production medium showed a biphasic response. At the highest tested concentration of $\mathrm{KH}_{2} \mathrm{PO}_{4}(200 \mathrm{mM})$, the maximum concentration of clavulanic acid dropped drastically to $524 \mathrm{mg} / \mathrm{L}$. Thus the optimum concentration of $\mathrm{KH}_{2} \mathrm{PO}_{4}$ for clavulanic acid production was determined to be $10 \mathrm{mM}$ (with a maximum clavulanic acid concentration recorded at $878 \mathrm{mg} / \mathrm{L}$ ).

Furthermore, the $\mathrm{pH}$ of fermentation media was described as having a profound effect on clavulanic acid yield (Saudagar and Singhal, 2007a; Salem-Berkhit et al., 2010). By using fermentation media with different $\mathrm{pH}$, different levels of clavulanic acid were seen; with maximum concentrations reported at $\mathrm{pH} 7$. Similar patterns of clavulanic acid production were seen in the tested fermentation media regardless of the sole carbon sources used (i.e., glycerol or olive oil). In addition, Costa and Badino (2012) reported that the temperature at which fermentation was carried out may lead to variation in clavulanic acid production. Low fermentation temperature $\left(20^{\circ} \mathrm{C}\right)$ resulted in maximum clavulanic acid concentration as high as $1266.2 \mathrm{mg} / \mathrm{L}$ as compared to $631.6 \mathrm{mg} / \mathrm{L}$ at $25^{\circ} \mathrm{C}$ and $168.7 \mathrm{mg} / \mathrm{L}$ at $30^{\circ} \mathrm{C}$.

Aeration or agitation speed throughout cultivation and production was described to affect clavulanic acid yield as well. In fact, the effect of aeration on the production of clavulanic acid can be tested with a direct experiment involving the use of the Erlenmeyer flask with different baffle heights (Lin et al., 2005). The results showed that the flask with a higher baffle height had a slight increase in the production of clavulanic acid $(180 \mathrm{mg} / \mathrm{L})$, as compared to a normal Erlenmeyer flask $(90 \mathrm{mg} / \mathrm{L})$. Another study showed that agitation speed has a positive correlation with clavulanic acid production (Rosa et al., 2005). At $800 \mathrm{rpm}$, two flasks with different oxygen flow rates showed similar levels of maximum clavulanic acid concentration, with $475 \mathrm{mg} / \mathrm{L}$ obtained from the flask with the lowoxygen flow rate and $482 \mathrm{mg} / \mathrm{L}$ 
from the flask with the low oxygen flow rate. A study by Cerri and Badino (2012) also supported the view that an increase in agitation speed leads to higher production of clavulanic acid, but not oxygen flow. When the oxygen flow was increased to $4.1 \mathrm{vvm}$, the maximum concentration of clavulanic acid was observed to be $269 \mathrm{mg} / \mathrm{L}$, which is approximately half of the maximum concentration observed with an oxygen flow of $3 \mathrm{vvm}(454 \mathrm{mg} / \mathrm{L})$. However, these results were contradictory with a previous study by Belmar-Beiny and Thomas (1991) which showed that there is no significant difference in clavulanic acid production as a result of different stirring speeds, even with same oxygen flow rate.

Besides that, the presence of redox-cycling agents in the production media may influence the production of clavulanic acid (Kwon and Kim, 1998). Five redox-cycling agents were tested-methyl viologen, menadione, plubmagin, phenazine methosulfate, and hydrogen peroxide $\left(\mathrm{H}_{2} \mathrm{O}_{2}\right)$. All of the redox-cycling agents promoted the production of clavulanic acid, except methyl viologen $(9.96-25.89 \mathrm{mg} / \mathrm{L})$. The highest maximum clavulanic acid concentration was described with phenazine methosulfate $(63.73-103.56 \mathrm{mg} / \mathrm{L})$, followed by plumbagin (55.76-79.66 mg/L), and $\mathrm{H}_{2} \mathrm{O}_{2}(51.78-69.71 \mathrm{mg} / \mathrm{L})$.

\section{Comparison between Batch and Fed-Batch Fermentations}

Among the selected studies, there were a total of seven fedbatch fermentation experiments, with the majority looking at the effect of adding glycerol into the fermenter over a period of time. Most of the studies took similar approaches to study the effect of glycerol in fed-batch fermentations: (a) by maintaining glycerol at a certain level throughout the fermentation period and/or (b) by adding fixed amounts of glycerol at fixed time points (Chen et al., 2002; Neto et al., 2005; Saudagar and Singhal, 2007b; Teodoro et al., 2010; Costa and Badino, 2012). Regardless of the methods, the study showed higher levels of maximum clavulanic acid concentration than control in the fed-batch fermentation. Comparing batch and fed-batch fermentation, fedbatch fermentation systems seemed to generate a higher yield of clavulanic acid (Chen et al., 2002; Neto et al., 2005; Bellão et al., 2013). A study by Bellão et al. (2013) observed a higher maximum clavulanic acid concentration in the latter method at $982.1 \mathrm{mg} / \mathrm{L}$, as compared to $348.5 \mathrm{mg} / \mathrm{L}$. Apart from the addition of glycerol, lower fermentation temperatures also resulted in a higher yield of clavulanic acid, which was also observed in both batch and fed-batch fermentation.

Besides that, the effects of amino acid was also studied using the fed-batch fermentation approach. Following batch fermentation that revealed increased clavulanic acid production by arginine and threonine, Saudagar and Singhal (2007b) showed that by using fed-batch fermentation technologies; the production of the compound could be further increased. Nevertheless, another study reported lower clavulanic acid yield in fed-batch fermentation with the addition of ornithine as compared to batch fermentation (Chen et al., 2003). On top of that, an increase in the amount of clavulanic acid produced was seen in other fed-batch experiments with the addition of glycerol and arginine. Chen et al. (2003) reported that fed-batch fermentation using glycerol, ornithine, and arginine; yielded different amounts of clavulanic acid, whereby the highest amount was demonstrated with glycerol $(300 \mathrm{mg} / \mathrm{L})$, followed by arginine $(210 \mathrm{mg} / \mathrm{L})$, and the lowest with ornithine $(110 \mathrm{mg} / \mathrm{L})$. The addition of glycerol together with either of the amino acids resulted in intermediate values, where the combination of glycerol and arginine produced $130 \mathrm{mg} / \mathrm{L}$; while glycerol and ornithine resulted in $200 \mathrm{mg} / \mathrm{L}$. Meanwhile, Teodoro et al. (2010) investigated the influence of the presence of ornithine in batch and fed-batch fermentation systems on clavulanic acid production. The study did not find any significant changes in maximum clavulanic acid production due to ornithine, regardless of batch or fed-batch fermentation systems. Interestingly, the same study also revealed an insignificant difference in maximum clavulanic acid concentration when the feeding media (which possesses the same composition as the production media) was replaced with distilled water containing only glycerol and ornithine (at same concentrations as the production media).

\section{DISCUSSION}

The microbial fermentation system is important for the discovery and development of pharmaceutical drugs. Clavulanic acid as a $\beta$-lactamase inhibitor was initially isolated from S. clavuligerus ATCC 27064 using the traditional fermentation system (Higgens and Kastner, 1971; Brown et al., 1976). $\beta$-lactamase inhibitors help to prevent drug resistance against $\beta$-lactam antibiotics, and allows successful eradication of harmful pathogens. Considering its therapeutic value against infectious diseases, the biosynthesis pathways of clavulanic acid in S. clavuligerus have been studied extensively over the years; beginning around 1980s by a research group led by Romero et al. (1986) (Figure 1). The two precursors involved in clavulanic acid biosynthesis, arginine and glyceraldehyde-3-phosphate; undergo a series of enzymatic processes to form the $\beta$-lactam inhibitor. Given that both arginine and glyceraldehyde-3-phosphate play an important role in primary metabolism, the production of clavulanic acid could be improved by refining the composition of the fermentation media (Kirk et al., 2000). It is also presumed that $S$. clavuligerus produces higher amount of clavulanic acid when there is adequate supply of these precursors. The wild type strain of S. clavuligerus is unable to metabolize glucose, and further molecular studies revealed that the strain lacks the expression of the glucose permease gene (Garcia-Dominguez et al., 1989; Pérez-Redondo et al., 2010).

Based on literature obtained in this study, glycerol was found to be the most popular choice of carbon source in clavulanic acid production; in order to ensure an efficient supply of glyceraldehyde-3-phosphate Once glycerol is metabolized into glyceraldehyde-3-phosphate, it can either enter the clavulanic acid biosynthesis pathway, or be involved in glycolytic or gluconeogenesis reactions (Figure 3). The inclusion of glycerol enhances the production of clavulanic acid as compared to carbohydrates, as glycerol provides a higher energy content on a weight-by weight basis (Efthimiou et al., 2008). As glycerol serves as a backbone for triglycerides, its utilization by S. clavuligerus has prompted researchers to study the potential of other oils 


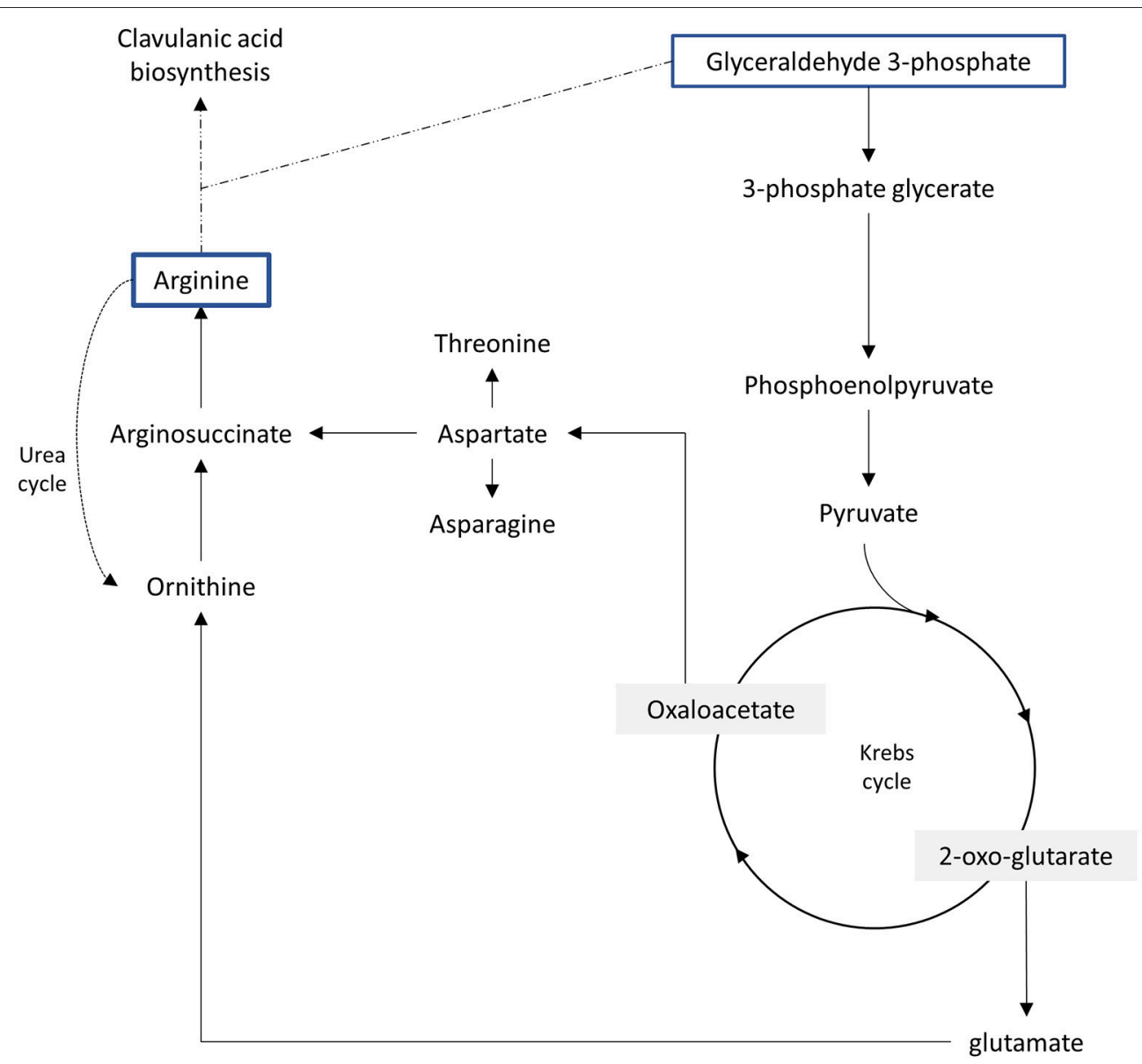

FIGURE 3 | Important pathways that could affect metabolic pool of clavulanic acid precursors - arginine and glutaraldehyde-3-phosphate (Kirk et al., 2000; Bushell et al., 2006).

to be used as a source of carbon. Vegetable oils such as olive oil and corn oil may serve as cost-effective options as they are readily available at a lower cost compared to carbohydrate substrates. As compared to glycerol, vegetable oils seem to be a more attractive source of energy; as a typical oil contains about 2.4 times more energy than glycerol (Stowell, 1987). Other than preventing carbon catabolite regulation, these oils could act as an antifoam, preventing the formation of foam in the media that may impede gas exchange and negatively affect bacteria growth (Friberg et al., 1989; Görke and Stülke, 2008). Overall, the utilization of vegetable oils might be a better choice for clavulanic acid production in fermentation processes compared to glycerol, as these natural oils may further enrich the media with the presence of various polyunsaturated fatty acids (e.g., oleic acid, linoleic acid) (Park et al., 1994). As the addition of olive oil has been shown to greatly improve clavulanic acid production, further investigations on the effect of vegetable oils could suggest the potential of these compounds to be exploited as a cheaper alternative source of carbon in fermentation processes.

On the other hand, many studies have shown the potential of arginine and ornithine as specific precursors for clavulanic acid. These two amino acids are believed to be interconvertible by the urea cycle via the enzymatic action of arginase (Figure 3). Even though presence of the urea cycle in prokaryotes was once considered unusual, several recent studies have reported arginase activity in S. clavuligerus (Mendz and Hazell, 1996; Bushell et al., 2006). These reports then further suggest an important role of this pathway in clavulanic acid biosynthesis. Thus, the addition of arginine or ornithine in fermentation media would increase the flow of C5 precursors into the biosynthesis pathway, which then subsequently increase the production of clavulanic acid (Romero et al., 1986; Lynch and Yang, 2004). From the included studies, one study showed that L-threonine improved the production of clavulanic acid (Saudagar and Singhal, 2007b). Indeed the supplementation of L-threonine was found to prevent the anaplerotic flux on pyruvate to synthesize amino acids such as isoleucine, which in turn increased the availability of C3 precursors and eventually enhanced clavulanic acid production (Ives and Bushell, 1997; Bushell et al., 2006; Saudagar and Singhal, 2007b). Even though the study by Saudagar and Singhal (2007b) has emphasized the role of L-threonine in clavulanic acid production, arginine and ornithine have demonstrated relatively strong influence on clavulanic acid production in S. clavuligerus. Thus, further studies on L-threonine could eventually shed 
some light on the importance of this amino acid in clavulanic acid production, particularly using the metabolic flux analysis approach.

Apart from media composition, environmental stress is known to affect the production of secondary metabolites in microorganisms including members of Streptomyces genus. The availability of oxygen determines the growth and survival of the bacteria as well as production of secondary metabolites, which includes antibiotics in S. clavuligerus (Yegneswaran et al., 1991). Out of the selected studies, there were three studies which highlighted the importance of oxygen control and agitation speed. Fermentation in bioreactors is often paired with computers for precise control of reaction conditions. Rosa et al. (2005) showed that the high speed stirring promoted the production of clavulanic acid, which was associated with a lower amount of biomass production. It is believed that the high speed stirring prevents cell clumping and also causes cell shearing (Toma et al., 1991; Rosa et al., 2005). Additionally, Kwon and Kim (1998) demonstrated that the addition of redox-cycling agents increased clavulanic acid production. The presence of reactive oxygen species in the fermentation media leads to an imbalance in redox status, which could trigger stress and damage microbials, or even lead to cell death (Cabiscol et al., 2010). The breakage and/or damage of bacterial cells upon exposure to such stress in turn, encourage the neighboring cells to produce secondary metabolites in an attempt to survive and protect against the challenge (Toma et al., 1991; Joshi et al., 1996; Rosa et al., 2005).

In addition, temperature and $\mathrm{pH}$ control are also crucial for the production of clavulanic acid and stability of the compound. S. clavuligerus ATCC 27064 was reported to have optimal growth from 26 to $30^{\circ} \mathrm{C}$ with no growth above $37^{\circ} \mathrm{C}$ (Higgens and Kastner, 1971), while most of the selected studies reported that fermentation temperature ranged between 20 and $30^{\circ} \mathrm{C}$. Costa and Badino (2012) demonstrated a maximum clavulanic acid concentration of $1266.2 \mathrm{mg} / \mathrm{L}$ when fermentation was performed at $20^{\circ} \mathrm{C}$. The study also mentioned that lower concentrations of clavulanic acid was observed with the increase in fermentation temperature. Even though a low temperature of $20^{\circ} \mathrm{C}$ was not favorable for the growth of $S$. clavuligerus, high production yield of clavulanic acid was observed. There are two possible explanations that could lead to this observation: (a) the low temperature places "cold" stress upon the organism which in turn promotes the production of secondary metabolites (including clavulanic acid); (b) the low temperature may have lowered degradation rate of clavulanic acid, thus ensuring the stability of the compound (Beales, 2004; Bersanetti et al., 2005; Jerzsele and Nagy, 2009; Santos et al., 2009; Feng et al., 2011). Similarly, $\mathrm{pH}$ of the medium could affect the growth of S. clavuligerus, as it is described to grow between $\mathrm{pH} 5.0$ and 8.5, however sporulation is not observed from $\mathrm{pH} 7.0$ to 8.5 (Higgens and Kastner, 1971). Hence, the determination of the optimum temperature and $\mathrm{pH}$ for the fermentation process is critical as slight changes in these factors could tip off the balance between the growth of the organism (biomass) and the production of secondary metabolite(s). On top of that, clavulanic acid was shown to be more stable at a neutral $\mathrm{pH}$, as the decomposition rate was described to be higher at acidic or alkaline $\mathrm{pH}$ (Jerzsele and Nagy, 2009). Taken altogether, the pH of the media and fermentation temperature may play important roles in clavulanic acid production as these factors could eventually lead to degradation of this valuable compound.

In this review, most of the selected papers used a onefactor-at-a-time method in batch fermentation to study the effect of carbon or nitrogen sources on clavulanic production in S. clavuligerus. However, two studies incorporated more complicated analyses in their studies to facilitate multifactorial comparisons. For instance, Wang et al. (2005) proposed using statistical methods to optimize the fermentation media for clavulanic acid production by $S$. clavuligerus. By combining factional factorial design and response surface methodology, the study suggested optimal concentration of three of the most important components identified via factional factorial designsoy meal powder $(38.102 \mathrm{~g} / \mathrm{L}), \mathrm{FeSO}_{4} .7 \mathrm{H}_{2} \mathrm{O}(0.395 \mathrm{~g} / \mathrm{L})$, and ornithine (1.177 g/L). Meanwhile, Saudagar and Singhal (2007a) designed a slightly different fermentation media by undertaking another statistical approach to optimize fermentation media for clavulanic acid production. By using the $\mathrm{L}_{25}$ orthogonal array method, the study suggested optimum concentrations of soybean flour $(8.8 \%)$, soybean oil $(1.2 \%)$, dextrin $(1.0 \%)$, yeast extract (1.5\%), and $\mathrm{KH}_{2} \mathrm{PO}_{4}(0.2 \%)$; with an optimal $\mathrm{pH}$ of 7.0 \pm 0.2 . Thus in designing a fermentation media for clavulanic acid production, it is important to ensure that the media can support the proper growth of S. clavuligerus; as well as provide factors that could stimulate the production of valuable secondary metabolites. Following media optimization, several studies have also incorporated another strategy to maximize the production of clavulanic acid-by utilizing fed-batch fermentation systems which represent a high throughput platform as compared to traditional batch fermentation methods.

The main difference between batch and fed-batch fermentation systems is that the latter is frequently monitored with the assistance of sophisticated technologies and allows precise control of the entire fermentation process (Longobardi, 1994; Li et al., 2014). As a scale-up production process, the fed-batch fermentation system often allows an increase in productivity with a concomitant decrease in production cost. At the time of writing, the current report is one of the first that reviews and investigates the effects of fermentation conditions affecting the production of clavulanic acid in S. clavuligerus; and further compares the batch and fed-batch fermentation systems for clavulanic acid production. Costa and Badino (2012) and Bellão et al. (2013) reported the utilization of glycerol in fed-batch fermentation systems resulted in a surge in the production of clavulanic acid (observed as a 1.2-2.8-fold increase in maximum clavulanic acid amount, depending on other factors such as temperature). Compared to glycerol, the addition of amino acid (as a source of nitrogen) in fed-batch fermentation showed less of an effect on the production of clavulanic acid. Only three studies reported the effect of amino acid in fed-batch fermentation (Chen et al., 2003; Saudagar and Singhal, 2007b; Teodoro et al., 2010); where one of the studies discovered that the addition of arginine and ornithine did not affect the production of clavulanic acid in both batch and fed-batch 
fermentation systems (Saudagar and Singhal, 2007b). Chen et al. (2003) discovered that ornithine increased the production of clavulanic acid in batch but not fed-batch fermentation, while arginine increased the production in fed-batch but not batch fermentation. Given that the fed-batch fermentation system entails a scale-up process, researchers would expect a higher yield of end-products (Modak et al., 1986; Thiry and Cingolani, 2002; Hewitt and Nienow, 2007). However, it has been suggested that sometimes a large-scale fed-batch fermentation may not generate similar results as observed in small-scale batch fermentation, as the fed-batch fermentation system involves a more dynamic environment (Hewitt and Nienow, 2007). Nevertheless, fedbatch fermentation appears to be a better fermentation strategy for clavulanic acid production as demonstrated in the selected studies. Likewise, further investigations using this fermentation method could upscale clavulanic acid production and ensure a better understanding of the biosynthesis pathways for this valuable compound.

\section{FUTURE PROSPECT AND CONCLUSION}

Over a span of 30 years, the research on the fermentation process for the production of clavulanic acid has gained remarkable interest from the scientific community. In this systematic review, a total of 29 studies was selected after a thorough literature search. It is worth mentioning that there were some inconsistencies in the measurement of clavulanic acid production in S. clavuligerus as some studies did not report the standard deviation even though the experiments were carried out in replicate. This then did not allow for results to be synthesized quantitatively and perform a meta-analysis. The majority of the articles highlighted the importance of media composition and supplements in the production of clavulanic acid, particularly glycerol, vegetable oils, and the amino acids, arginine and ornithine. In batch fermentation systems which are commonly used for laboratoryscale production, the utilization of various sugars (e.g., dextrin and sucrose) and glycerol as sole carbon sources in clavulanic acid production requires further investigation; as current studies have reported inconsistent results and the role of these compounds in the biosynthesis pathways is yet to be clearly defined. Further investigation into the role of carbohydrates and glycerol in clavulanic acid biosynthesis would greatly improve the

\section{REFERENCES}

Ara, I., Bukhari, N. A., Aref, N. M., Shinwari, M. M., and Bakir, M. A. (2014). Antiviral activities of streptomycetes against tobacco mosaic virus (TMV) in Datura plant: Evaluation of different organic compounds in their metabolites. Afr. J. Proc. Natl. Acad. Sci. U.S.A. 11, 2130-2138. doi: 10.5897/AJB11. 3388

Aharonowitz, Y., and Demain, A. L. (1978). Carbon catabolite regulation of cephalosporin production in Streptomyces clavuligerus. Antimicrobial Agents Chemother. 14, 159-164.

Azman, A. S., Othman, I., Velu, S. S., Chan, K. G., and Lee, L. H. (2015). Mangrove rare actinobacteria: taxonomy, natural compound, and discovery of bioactivity. Front. Microbiol. 6:856. doi: 10.3389/fmicb.2015.00856 knowledge of media optimization. Nevertheless, the utilization of different oil sources as a sole carbon source and amino acids as a source of nitrogen in the fermentation media seems to have a strong influence on clavulanic acid production in S. clavuligerus; followed by other factors such as $\mathrm{pH}$ and temperature. Among the vegetable oils, media supplemented with olive oil showed the highest level of clavulanic acid production, which indicates that olive oil contains potentially important nutrients that could improve the production of the antibiotics. Furthermore, amino acids such as arginine and ornithine which could serve as C5 precursors, have also been shown to increase clavulanic acid yield. For the most part, the development of scale-up production tools such as fed-batch fermentation systems could offer a "budget-friendly" method for clavulanic acid production, as this is particularly important for the pharmaceutical industry where production cost is one of the major concerns. With the advancement of next generation sequencing technologies, researchers have identified numerous genes involved in clavulanic acid biosynthesis in S. clavuligerus including the clavaminate synthases genes (Medema et al., 2011). By combining this knowledge, further studies involve scale-up productions would be beneficial to identify biosynthetic roles, as well as determine the regulation of these carbon and nitrogen sources in clavulanic acid production in S. clavuligerus.

\section{AUTHOR CONTRIBUTIONS}

H-LS and JW-FL contributed to the literature database search, data collection, data extraction, data analysis and writing of the manuscript. H-LS, JW-FL, NC, SAJ, UDP, K-GC, B-HG and LHL performed data analysis and rationalization of the results. The topic was conceptualized by B-HG and L-HL.

\section{ACKNOWLEDGMENTS}

This work was supported by a University of Malaya for High Impact Research Grant (UM-MOHE HIR Nature Microbiome Grant No. H-50001-A000027 and No. A000001-50001) awarded to K.-G. C., MOSTI eScience Funds (06-02-10-SF0300) awarded to L.-H. L. and (02-02-10-SF0215) awarded to B.-H.G, External Industry Grant (Biotek Abadi - Vote No. GBA-808138 \& GBA808813) awarded to L.-H.L.
Beales, N. (2004). Adaptation of microorganisms to cold temperatures, weak acid preservatives, low $\mathrm{pH}$, and osmotic stress: a review. Comp. Rev. Food Sci. Food Safety 3, 1-20. doi: 10.1111/j.1541-4337.2004.tb00057.x

Bellão, C., Antonio, T., Araujo, M. L. G. C., and Badino, A. C. (2013). Production of clavulanic acid and cephamycin C by Streptomyces clavuligerus under different fed-batch conditions. Braz. J. Chem. Engin. 30, 257-266. doi: 10.1590/S010466322013000200004

Belmar-Beiny, M. T., and Thomas, C. R. (1991). Morphology and clavulanic acid production of Streptomyces clavuligerus: effect of stirrer speed in batch fermentations. Biotech. Bioengin. 37, 456-462. doi: 10.1002/bit. 260370507

Bérdy, J. (2005). Bioactive microbial metabolites. J. Antibiotics 58, 1-26. doi: 10.1038/ja.2005.1 
Bersanetti, P. A., Almeida, R. M., Barboza, M., Araújo, M. L. G., and Hokka, C. O. (2005). Kinetic studies on clavulanic acid degradation. Biochem. Engin. J. 23, 31-36. doi: 10.1016/j.bej.2004.10.007

Brown, A. G., Butterworth, D., Cole, M., Hanscomb, G., Hood, J. D., Reading, C., et al. (1976). Naturally-occurring beta-lactamase inhibitors with antibacterial activity. J. Antibiotics 29, 668-669. doi: 10.7164/antibiotics.29.668

Bushell, M. E., Kirk, S., Zhao, H. J., and Avignone-Rossa, C. A. (2006). Manipulation of the physiology of clavulanic acid biosynthesis with the aid of metabolic flux analysis. Proc. Natl. Acad. Sci. U.S.A. 39, 149-157. doi: 10.1016/j.enzmictec.2006.01.017

Cabiscol, E., Tamarit, J., and Ros, J. (2010). Oxidative stress in bacteria and protein damage by reactive oxygen species. Int. Microbiol. 3, 3-8.

Cerri, M. O., and Badino, A. C. (2012). Shear conditions in clavulanic acid production by Streptomyces clavuligerus in stirred tank and airlift bioreactors. Bioprocess Biosyst. Engin. 35, 977-984. doi: 10.1007/s00449-012-0682-8

Chen, K. C., Lin, Y. H., Tsai, C. M., Hsieh, C. H., and Houng, J. Y. (2002). Optimization of glycerol feeding for clavulanic acid production by Streptomyces clavuligerus with glycerol feeding. Biotech. Lett. 24, 455-458. doi: 10.1023/A:1014553109425

Chen, K. C., Lin, Y. H., Wu, J. Y., and Hwang, S. C. J. (2003). Enhancement of clavulanic acid production in Streptomyces clavuligerus with ornithine feeding. Enzyme Microbial. Tech. 32, 152-156. doi: 10.1016/S0141-0229(02)00280-6

Costa, C. L., and Badino, A. C. (2012). Production of clavulanic acid by Streptomyces clavuligerus in batch cultures without and with glycerol pulses under different temperature conditions. Biochem. Engin. J. 69, 1-7. doi: 10.1016/j.bej.2012.08.005

Demain, A. L. (1999). Pharmaceutically active secondary metabolites of microorganisms. Appl. Microbial. Biotech. 52, 455-463. doi: $10.1007 / \mathrm{s} 002530051546$

Demain, A. L., and Sanchez, S. (2009). Microbial drug discovery: 80 years of progress. J. Antibiotics 62, 5-16. doi:10.1038/ja.2008.16

de Lima Procópio, R. E., da Silva, I. R., Martins, M. K., de Azevedo, J. L., and de Araújo, J. M. (2012). Antibiotics produced by Streptomyces. Braz. J. Infect. Dis. 16, 466-471. doi: 10.1016/j.bjid.2012.08.014

Efthimiou, G., Thumser, A. E., and Avignone-Rossa, C. A. (2008). A novel finding that Streptomyces clavuligerus can produce the antibiotic clavulanic acid using olive oil as a sole carbon source. J. Appl. Microbiol. 105, 2058-2064. doi: 10.1111/j.1365-2672.2008.03975.x

Feng, W. H., Mao, X. M., Liu, Z. H., and Li, Y. Q. (2011). The ECF sigma factor SigT regulates actinorhodin production in response to nitrogen stress in Streptomyces coelicolor. Appl. Microbiol. Biotech. 92, 1009-1021. doi: 10.1007/s00253-011-3619-2

Friberg, S. E., Blute, I., and Stenius, P. (1989). Foam stability in a glycerol system. J. Colloid Interface Sci. 127, 573-582. doi: 10.1016/0021-9797(89) 90062-3

Garcia-Dominguez, M., Martin, J. F., and Liras, P. (1989). Characterization of sugar uptake in wild-type Streptomyces clavuligerus, which is impaired in glucose uptake, and in a glucose-utilizing mutant. J. Bacteriol. 171, 6808-6814.

Görke, B., and Stülke, J. (2008). Carbon catabolite repression in bacteria: many ways to make the most out of nutrients. Nat. Rev. Microbiol. 6, 613-624. doi: 10.1038/nrmicro1932

Gouveia, E. R., Baptista-Neto, A., Azevedo, A. G., Badino, A. C., and Hokka, C. O. (1999). Improvement of clavulanic acid production by Streptomyces clavuligerus in medium containing soybean derivatives. World J. Microbiol. Biotech. 15, 623-627. doi: 10.1023/A:1008942405378

Gouveia, E. R., Baptista-Neto, A., Badino, A. C. Jr., and Hokka, C. O. (2001). Optimisation of medium composition for clavulanic acid production by Streptomyces clavuligerus. Biotech. Lett. 23, 157-161. doi: 10.1023/A:1010356231673

Hewitt, C. J., and Nienow, A. W. (2007). The scale-up of microbial batch and fed-batch fermentation processes. Adv. Appl. Microbial. 62, 105-135. doi: 10.1016/S0065-2164(07)62005-X

Higgens, C. E., and Kastner, R. E. (1971). Streptomyces clavuligerus sp. nov., a $\beta$-lactam antibiotic producer. Int. J. Syst. Evol. Microbiol. 21, 326-331. doi: 10.1099/00207713-21-4-326

Ives, P. R., and Bushell, M. E. (1997). Manipulation of the physiology of clavulanic acid production in Streptomyces clavuligerus. Microbiology 143, 3573-3579. doi: 10.1099/00221287-143-11-3573
Jerzsele, Á., and Nagy, G. (2009). The stability of amoxicillin trihydrate and potassium clavulanate combination in aqueous solutions. Acta Vet. Hung. 57, 485-493. doi: 10.1556/AVet.57.2009.4.3

Joshi, J. B., Elias, C. B., and Patole, M. S. (1996). Role of hydrodynamic shear in the cultivation of animal, plant and microbial cells. Chem. Engin. J. Biochem. Engin. J. 62, 121-141. doi: 10.1016/0923-0467(95)03062-X

Kanehisa, M., and Goto, S. (2000). KEGG: kyoto encyclopedia of genes and genomes. Nucleic Acids Res. 28, 27-30. doi: 10.1093/nar/28.1.27

Kanehisa, M., Sato, Y., Kawashima, M., Furumichi, M., and Tanabe, M. (2016). KEGG as a reference resource for gene and protein annotation. Nucleic Acids Res. 44, D457-D462. doi: 10.1093/nar/gkv1070

Kim, S. J., Kim, J. O., Shin, C. H., Park, H. W., and Kim, C. W. (2009). An approach to strain improvement and enhanced production of clavulanic acid in Streptomyces clavuligerus. Biosci. Biotech. Biochem. 73, 160-164. doi: 10.1271/bbb. 80569

Kirk, S., Avignone-Rossa, C. A., and Bushell, M. E. (2000). Growth limiting substrate affects antibiotic production and associated metabolic fluxes in Streptomyces clavuligerus. Biotech. Lett. 22, 1803-1809. doi: 10.1023/A:1005670603596

Kwon, H. J., and Kim, S. U. (1998). Enhanced biosynthesis of clavulanic acid in Streptomyces clavuligerus due to oxidative challenge by redox-cycling agents. Appl. Microbiol. Biotechnol. 49, 77-83. doi: 10.1007/s002530051140

Lebrihi, A., Germain, P., and Lefebvre, G. (1987). Phosphate repression of cephamycin and clavulanic acid production by Streptomyces clavuligerus. Appl. Microbial. Biotech. 26, 130-135. doi: 10.1007/BF00253896s

Lee, L.-H., Zainal, N., Azman, A.-S., Eng, S.-K., Ab Mutalib, N.-S., Yin, W.F., et al. (2014a). Streptomyces pluripotens sp. nov., a bacteriocin-producing streptomycete that inhibits meticillin-resistant Staphylococcus aureus. Int. J. Syst. Evol. Microbiol. 64, 3297-3306. doi: 10.1099/ijs.0.065045-0

Lee, L.-H., Zainal, N., Azman, A.-S., Eng, S.-K., Goh, B.-H., Yin, W.-F., et al. (2014b). Diversity and antimicrobial activities of actinobacteria isolated from tropical mangrove sediments in Malaysia. Sci. World J. 2014, 1-14. doi: $10.1155 / 2014 / 698178$

Lee, P. C., and Ho, C. C. (1996). Production of clavulanic acid and cephamycin C by Streptomyces clavuligerus in palm-oil medium. World J. Microbiol. Biotech. 12, 73-75. doi: 10.1007/BF00327805

Li, T., Chen, X. B., Chen, J. C., Wu, Q., and Chen, G. Q. (2014). Open and continuous fermentation: products, conditions and bioprocess economy. Biotech. J. 9, 1503-1511. doi: 10.1002/biot.201400084

Lin, Y. H., Hwang, S. C. J., Gong, J. T., Wu, J. Y., and Chen, K. C. (2005). Using redox potential to detect microbial activities during clavulanic acid biosynthesis in Streptomyces clavuligerus. Biotech. Lett. 27, 1791-1795. doi: 10.1007/s10529-005-3727-0

Longobardi, G. P. (1994). Fed-batch versus batch fermentation. Bioprocess Biosyst. Eng. 10, 185-194. doi: 10.1007/BF00369529

Lynch, H. C., and Yang, Y. (2004). Degradation products of clavulanic acid promote clavulanic acid production in cultures of Streptomyces clavuligerus. Enzyme Microbial Tech. 34, 48-54. doi: 10.1016/j.enzmictec.2003.08.003

Maranesi, G. L., Baptista-Neto, A., Hokka, C. O., and Badino, A. C. (2005). Utilization of vegetable oil in the production of clavulanic acid by Streptomyces clavuligerus ATCC 27064. World J. Microbiol. Biotech. 21. 509-514. doi: $10.1007 /$ s11274-004-2393-z

Manivasagan, P., Venkatesan, J., Sivakumar, K., and Kim, S. K. (2014). Pharmaceutically active secondary metabolites of marine actinobacteria. Microbiol. Res. 169, 262-278. doi: 10.1016/j.micres.2013.07.014

Medema, M. H., Alam, M. T., Heijne, W. H., van den Berg, M. A., Müller, U., Trefzer, A., et al. (2011). Genome-wide gene expression changes in an industrial clavulanic acid overproduction strain of Streptomyces clavuligerus. Microbial Biotech. 4, 300-305. doi: 10.1111/j.1751-7915.2010.00226.x

Mendz, G. L., and Hazell, S. L. (1996). The urea cycle of Helicobacter pylori. Microbiology 142, 2959-2967. doi: 10.1099/13500872-142-10-2959

Modak, J. M., Lim, H. C., and Tayeb, Y. J. (1986). General characteristics of optimal feed rate profiles for various fed-batch fermentation processes. Biotech. Bioengin. 28, 1396-1407. doi: 10.1002/bit.260280914

Moher, D., Liberati, A., Tetzlaff, J., Altman, D. G., and The PRISMA Group (2009). Preferred reporting items for systematic reviews and meta-analyses: the PRISMA statement. PLoS Med. 6:e1000097. doi: 10.1371/journal.pmed 1000097 
Neto, A. B., Hirata, D. B., Cassiano Filho, L. C. M., Bellão, C., Badino Júnior, A. C., and Hokka, C. O. (2005). A study on clavulanic acid production by Streptomyces clavuligerus in batch, fed-batch and continuous processes. Braz. J. Chem. Engin. 22, 557-563. doi: 10.1590/S0104-66322005000 400008

Newman, D. J., Cragg, G. M., and Snader, K. M. (2000). The influence of natural products upon drug discovery. Nat. Prod. Rep. 17, 215-234. doi: 10.1039/A902202C

Okamoto, R., Kiyoshima, K., Yamamoto, M., Takada, K., Ohnuki, T., Ishikura, T., et al. (1982). New macrolide antibiotics produced by mutants from Streptomyces fradiae NRRL 2702. J. Antibiotics 35, 921-924. doi: 10.7164/antibiotics.35.921

Ortiz, S. C. A., Hokka, C. O., and Badino, A. C. (2007). Utilization of soybean derivatives on clavulanic acid production by Streptomyces clavuligerus. Enzyme Microbial Tech. 4, 1071-1077. doi: 10.1016/j.enzmictec.2006.08.009

Page, M. G. (2012). "Beta-lactam antibiotics," in Antibiotic Discovery and Development, eds T. J. Dougherty and M. J. Pucci (New York, NY: Springer), 79-117.

Pérez-Redondo, R., Santamarta, I., Bovenberg, R., Martín, J. F., and Liras, P. (2010). The enigmatic lack of glucose utilization in Streptomyces clavuligerus is due to inefficient expression of the glucose permease gene. Microbiology 156, 1527-1537. doi: 10.1099/mic.0.035840-0

Park, Y. S., Momose, I., Tsunoda, K., and Okabe, M. (1994). Enhancement of cephamycin C production using soybean oil as the sole carbon source. Appl. Microbiol. Biotech. 40, 773-779. doi: 10.1007/BF00173973

Reading, C., and Cole, M. (1977). Clavulanic acid: a beta-lactamase-inhibiting beta-lactam from Streptomyces clavuligerus. Antimicrobial Agents Chemother. 11, 852-857. doi: 10.1128/AAC.11.5.852

Romero, J., Liras, P., and Martín, J. F. (1986). Utilization of ornithine and arginine as specific precursors of clavulanic acid. Appl. Environ. Microbiol. 52, 892-897.

Rosa, J. C., Neto, A. B., Hokka, C. O., and Badino, A. C. (2005). Influence of dissolved oxygen and shear conditions on clavulanic acid production by Streptomyces clavuligerus. Bioprocess Biosyst. Engin. 27, 99-104. doi: 10.1007/s00449-004-0386-9

Salem-Berkhit, M. M., Alanazi, F. K., and Alsarra, I. A. (2010). Improvement and enhancement of clavulanic acid production in Streptomyces clavuligerus using vegetable oils. Afr. J. Biotech. 9, 6806-6812. doi: 10.5897/AJB10.965

Santos, V. C., Pereira, J. F. B., Haga, R. B., Rangel-Yagui, C. O., Teixeira, J. A. C., Converti, A., et al. (2009). Stability of clavulanic acid under variable $\mathrm{pH}$, ionic strength and temperature conditions. A new kinetic approach. Biochem. Engin. J. 45, 89-93. doi: 10.1016/j.bej.2009.02.013

Saudagar, P. S., and Singhal, R. S. (2007a). A statistical approach using L25 orthogonal array method to study fermentative production of clavulanic acid by Streptomyces clavuligerus MTCC 1142. Appl. Biochem. Biotech. 136, 345-359. doi: $10.1007 / \mathrm{s} 12010-007-9030-\mathrm{x}$

Saudagar, P. S., and Singhal, R. S. (2007b). Optimization of nutritional requirements and feeding strategies for clavulanic acid production by Streptomyces clavuligerus. Bioresource Tech. 98, 2010-2017. doi: 10.1016/j.biortech.2006.08.003

Saudagar, P. S., Survase, S. A., and Singhal, R. S. (2008). Clavulanic acid: a review. Biotech. Adv. 26, 335-351. doi: 10.1016/j.biotechadv.2008.03.002

Schmidt, F. R. (2005). Optimization and scale up of industrial fermentation processes. Appl. Microbiol. Biotech. 68, 425-435. doi: 10.1007/s00253-0050003-0

Ser, H.-L., Palanisamy, U. D., Yin, W.-F., Abd Malek, S. N., Chan, K.-G., Goh, B.H., et al. (2015a). Presence of antioxidative agent, Pyrrolo[1,2-a]pyrazine-1,4dione,hexahydro- in newly isolated Streptomyces mangrovisoli sp. nov. Front. in Microbiol. 6:854. doi: 10.3389/fmicb.2015.00854
Ser, H.-L., Ab Mutalib, N.-S., Yin, W.-F., Chan, K.-G., Goh, B. H., and Lee, L. H. (2015b). Evaluation of antioxidative and cytotoxic activities of Streptomyces pluripotens MUSC 137 isolated from mangrove soil in Malaysia. Front. Microbiol. 6:1398. doi: 10.3389/fmicb.2015.01398

Stowell, J. D. (1987). "The application of oils and fats in antibiotic processes," in Carbon Substrates in Biotechnology, eds J. D. Stowell, A. J. Beardsmore, C. W. Keevil, and J. R. Woodward (Oxford: IRL Press), 139-159.

Teodoro, J. C., Baptista-Neto, A., Cruz-Hernández, I. L., Hokka, C. O., and Badino, A. C. (2006). Influence of feeding conditions on clavulanic acid production in fed-batch cultivation with medium containing glycerol. Appl. Microbial. Biotech. 72, 450-455. doi: 10.1007/s00253-005-0273-6

Teodoro, J. C., Baptista-Neto, A., Araujo, M. L. G. D. C., Hokka, C. O., and Badino, A. C. (2010). Influence of glycerol and ornithine feeding on clavulanic acid production by Streptomyces clavuligerus. Braz. J. Chem. Engin. 27, 499-506. doi: 10.1590/S0104-66322010000400001

Tan, L. T. H., Ser, H. L., Yin, W. F., Chan, K. G., Lee, L. H., and Goh, B. H. (2015). Investigation of antioxidative and anticancer potentials of Streptomyces sp. MUM256 isolated from Malaysia mangrove soil. Front. Microbial. 6:1316. doi: 10.3389/fmicb.2015.01316

Thakur, R., Roy, M. K., Dutta, N. N., and Bezbaruah, R. L. (1999). Coordinate production of cephamycin $\mathrm{c}$ and clavulanic acid by Streptomyces clavuligerus. Ind. J. Exp. Biol. 37, 1031-1033.

Thiry, M., and Cingolani, D. (2002). Optimizing scale-up fermentation processes. Trends Biotech. 20, 103-105. doi: 10.1016/S0167-7799(02)01913-3

Toma, M. K., Ruklisha, M. P., Vanags, J. J., Zeltina, M. O., Lelte, M. P., Galinine, N. I., et al. (1991). Inhibition of microbial growth and metabolism by excess turbulence. Biotech. Bioengin. 38, 552-556. doi: 10.1002/bit.260380514

Toussaint, K. A., and Gallagher, J. C. (2015). $\beta$-lactam/ $\beta$-lactamase inhibitor combinations from then to now. Ann. Pharmacother. 49, 86-98. doi: $10.1177 / 1060028014556652$

Waksman, S. A., Bugie, E., and Schatz, A. (1944). Isolation of antibiotic substances from soil micro-organisms, with special reference to streptothricin and streptomycin. Proc. Staff Meetings Mayo Clinic 19, 536-548.

Wang, Y. H., Yang, B., Ren, J., Dong, M. L., Liang, D., and Xu, A. L. (2005). Optimization of medium composition for the production of clavulanic acid by Streptomyces clavuligerus. Process Biochem. 40, 1161-1166. doi: 10.1016/j.procbio.2004.04.010

WHO. (2015). WHO Model List of Essential Medicines: 19th list (updated) April 2015.

Wilke, M. S., Lovering, A. L., and Strynadka, N. C. (2005). $\beta$-Lactam antibiotic resistance: a current structural perspective. Curr. Op. Microbial. 8, 525-533. doi: 10.1016/j.mib.2005.08.016

Yegneswaran, P. K., Gray, M. R., and Thompson, B. G. (1991). Effect of dissolved oxygen control on growth and antibiotic production in Streptomyces clavuligerus fermentations. Biotech. Prog. 7, 246-250. doi: 10.1021/bp00009a008

Conflict of Interest Statement: The authors declare that the research was conducted in the absence of any commercial or financial relationships that could be construed as a potential conflict of interest.

Copyright (c) 2016 Ser, Law, Chaiyakunapruk, Jacob, Palanisamy, Chan, Goh and Lee. This is an open-access article distributed under the terms of the Creative Commons Attribution License (CC BY). The use, distribution or reproduction in other forums is permitted, provided the original author(s) or licensor are credited and that the original publication in this journal is cited, in accordance with accepted academic practice. No use, distribution or reproduction is permitted which does not comply with these terms. 